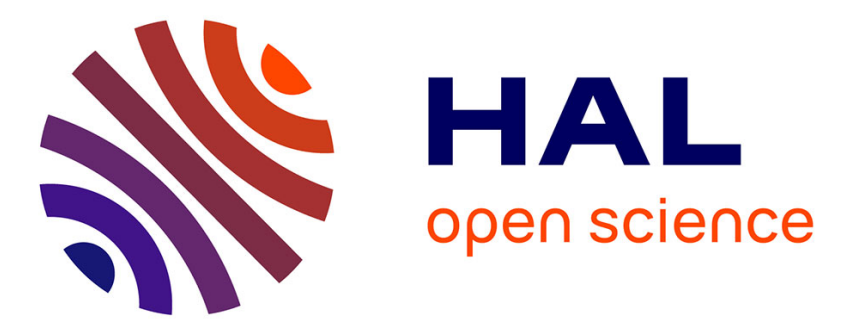

\title{
Fractional rheology of muscle precursor cells
}

\author{
E. Gerasimova-Chechkina, L. Streppa, L. Schaeffer, A. Devin, P. Argoul, A.
}

Arneodo, Françoise Argoul

\section{To cite this version:}

E. Gerasimova-Chechkina, L. Streppa, L. Schaeffer, A. Devin, P. Argoul, et al.. Fractional rheology of muscle precursor cells. Journal of Rheology, 2018, 62 (6), pp.1347 - 1362. 10.1122/1.5035127 . hal-01910887v2

\section{HAL Id: hal-01910887 \\ https://hal.science/hal-01910887v2}

Submitted on 15 Nov 2018 (v2), last revised 1 Jun 2021 (v3)

HAL is a multi-disciplinary open access archive for the deposit and dissemination of scientific research documents, whether they are published or not. The documents may come from teaching and research institutions in France or abroad, or from public or private research centers.
L'archive ouverte pluridisciplinaire HAL, est destinée au dépôt et à la diffusion de documents scientifiques de niveau recherche, publiés ou non, émanant des établissements d'enseignement et de recherche français ou étrangers, des laboratoires publics ou privés.

\section{(1)(1) $\$(0)$}

Distributed under a Creative Commons Attribution - NonCommercial - ShareAlikel 4.0 


\title{
Fractional rheology of muscle precursor cells
}

\author{
E. Gerasimova-Chechkina, ${ }^{1}$ L. Streppa, ${ }^{2,3}$ L. Schaeffer ${ }^{4}$ A. Devin, ${ }^{5}$ P. Argoul,,${ }^{6}$ A. Arneodo,,${ }^{2,3,7}$ and \\ F. Argoul ${ }^{2,3,7, a)}$ \\ ${ }^{1}$ Laboratory of Physical Foundation of Strength, Institute of Continuous Media Mechanics UB RAS, Perm, Russia \\ ${ }^{2}$ CNRS, UMR5672, Laboratoire de Physique, Ecole Normale Supérieure de Lyon, 69007 Lyon, France \\ ${ }^{3}$ Université de Lyon 1, 69100 Villeurbanne, France \\ ${ }^{4}$ CNRS UMR5239, Laboratoire de Biologie Moléculaire de la Cellule, Ecole Normale Supérieure de Lyon, 69007 Lyon, \\ France \\ ${ }^{5}$ CNRS UMR5095, Institut de Biochimie et de Génétique Cellulaire, Université de Bordeaux, 33077 Bordeaux, France \\ ${ }^{6}$ Université Paris-Est, Laboratoire EMGCU-MAST, IFSTTAR, 77447 Marne-la-Vallée Cedex 2, France \\ ${ }^{7}$ CNRS UMR5798, Laboratoire Ondes et Matière d'Aquitaine, Université de Bordeaux, 33405 Talence, France
}

\begin{abstract}
The authors propose a wavelet based decomposition of creep fluctuation signals recorded from living muscle precursor cells that revisit the traditional computation of their power spectrum from FFT based decomposition. This decomposition offers a higher sensitivity for detecting the occurrence of fractional fluctuations and for quantitatively estimating the power law exponent $\beta$ of this spectrum as a signature of the scale invariant rheology of living cells. This new method has also the unprecedented advantage of providing a test of the validity of the com monly assumed "monofractal" self similar (as compared to "multifractal" intermittent) nature of these fluctuations and hence accrediting the use of a single rheological exponent $\alpha=\beta / 2$. We report and discuss results obtained when applying this method to creep experiments per formed with an AFM nanoindenter placed in contact with single myoblasts and myotubes, adherent on collagen coated coverslips, and in dif ferent culture conditions.
\end{abstract}

\section{INTRODUCTION}

Eukaryotic cells are constructed from three sets of protein filaments arranged in a cross-linked cytoskeleton (CSK) network involving active and passive cross-linkers $[1,2]$. The actin-myosin II association constitutes the main engine for the muscular function of individual cells, and for their spatiotemporal dynamics such as cell contractility, adhesion, and motility $[3,4]$. Single cell rheology has been thoroughly investigated in the past few decades with a great diversity of experimental approaches, both in temporal and spectral domains [1,5 13]. Complex quantities such as the relaxation modulus $\widehat{G}(f)$ and the compliance $\widehat{J}(f)$ were estimated over five decades of $f$ from $10^{3} \mathrm{~Hz}$ to $100 \mathrm{kHz}$. At high frequencies $\left(f \geq 10^{3} \mathrm{~Hz}\right)$, the effective rheology of the actomyosin CSK was shown to be dominated by the mechanical and thermal properties of individual actin filaments or bundles $[1,2,14,15]$. At lower frequencies, in the $110^{3} \mathrm{~Hz}$ range, unbinding and rebinding events of passive cross-linkers make the CSK resemble a viscoelastic soft glassy material with a power-law (fractional) rheology $[5,79,16]$. In that frequency range, an indivisible relationship exists between storage (real part) and loss (imaginary part) components of $\widehat{G}(f)$ that both scale as $G^{\prime}(f) \sim G^{\prime \prime}(f) \sim f^{\alpha}$. The exponent $\alpha$ depends on the internal structure of the cell and its interplay with its

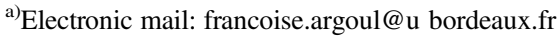

environment. For example, cells prestressed by strong adherence on stiff supports turn progressively to a more solid and elastic material $(\alpha \rightarrow 0)[4,9]$.

The mechanics of lipid layers forming artificial and cytoplasmic membranes was also reported to present quite interesting fractional hereditary rheology $[17,18]$. In physiological situations, the membrane stress is neither homogeneous spatially nor invariant in time. Beyond some stress threshold, the membrane may break or switch to a ceramide phase [17]. Actually, in living cells the mechanics of this lipid bilayer is not independent of the underlying actin cortex (actin-based filaments network). Its anchoring to the plasma membrane and its thickness (ranging from 0.05 to $1 \mu \mathrm{m}$ ) are maintained dynamically by the assistance of ATP driven molecular motors [bivalent cross-linking myosins (I) with lipid binding motifs and actin binding motifs]. This actin cortex is an isotropic network of actin filaments, oriented parallel to the plasma membrane with mesh size from 20 to $250 \mathrm{~nm}$ [19], facilitating the penetration of the cantilever tip (tip radius curvature $<10 \mathrm{~nm}$ ).

The fractional exponent $\alpha$ was experimentally estimated using either (i) forced periodic driving by an indenter in contact with the cell or (ii) "unforced" (thermal fluctuations) driving of small particles anchored to or embedded inside the cell [5 10]. This latter method is by far quicker since it allows one to explore a wide range of frequencies from a single recording. It becomes mandatory when the cell state evolves in time. However, it requires a frequency (spectral) 
decomposition of the recorded signal and in some situations a time-frequency decomposition. Most previous experimental studies relied on the Generalised Stokes Einstein Relation (GSER) to uncover, from thermal fluctuations, the complex viscoelastic modulus $\widehat{G}(f)[20,21]$. But the validity of GSER is still a debated issue since a living cell is an active glassy material that cannot be considered at thermal equilibrium [22 25]. Indeed, micro-tracers perceive an effective temperature due to the nonhomogeneous and nonstationary out-of-equilibrium properties of this material. Thus, a violation of the GSER is likely to manifest as a departure (e.g., anomalous intermittent multifractal diffusion [26]) from selfsimilar Gaussian statistics of tracer random fluctuations [27].

Atomic force microscopy (AFM) became rapidly an alternative to micro-particle trajectory tracking, because it could be used to periodically force the cantilever nanotip in contact with the tested material [13,28 30]. Recently, "unforced" microrheology of soft gels and cells was also achieved with AFM [11,31,32]. The advantage of AFM forcing, as compared to micro-particle tracing, is that except its nanotip in contact with the soft sample, the AFM cantilever body is outside the sample and is excited by the surrounding thermal chamber (at equilibrium). We can, therefore, reasonably identify the fluctuating force that drives randomly an AFM cantilever inside a liquid chamber as a Gaussian stochastic perturbation. For this experimental demonstration, we use muscle precursor cells (myoblasts and myotubes from a mouse model $\mathrm{C} 2 \mathrm{C} 12$ and human myoblasts) in different culture conditions: (i) normal, (ii) with an inhibition of the myosin function, and (iii) with a depletion of the source of energy (ATP) of the cell. We also study dystrophic (Duchenne muscular dystrophy DMD) human myoblasts as a comparative pathological situation. In this study, the creep experiments were performed for a constant loading force of $1 \mathrm{nN}$, which corresponds to a depth of penetration of the tip of the cantilever of 1 to $2 \mu \mathrm{m}$, depending on the rigidity of the cell. The typical shear modulus of these cells varies from 1 to $2 \mathrm{kPa}$ [13]. However, the tension of the (plasma membrane/actin-cortex) system was estimated to be $\sim 10{ }^{4} \mathrm{~N} / \mathrm{m}$ [33], which is about three orders of magnitude smaller than the nominal spring constant of the cantilever beam used in this work $(\sim 0.1 \mathrm{~N} / \mathrm{m})$. Hence, the cantilever is very stiff and during its penetration into the cell (prior to recording the cantilever deflection signal), it more likely perforates the plasma membrane, creating a local pore that may facilitate $\mathrm{Ca}^{2+}$ ion import into the cytoplasm [13].

The wavelet transform is a mathematical time-frequency (time-scale) decomposition of signals introduced in the early 1980s [34], which was applied to a great variety of situations in physics, physical chemistry, biology, signal and image processing, material engineering, mechanics, economics, epidemics, etc. [35 45]. Real experimental signals are very often nonstationary (they contain transient components), and involve a rather wide range of frequencies which may also drift in time. Standard Fourier analysis is, therefore, inadequate in these situations since it provides only statistical information about the relative contributions of the frequencies involved in the analyzed signal. The possibility to perform simultaneously a temporal and frequency decomposition of a given signal was first proposed by Gabor for the theory of communication [46]. Later on, two distinct approaches (based on different wavelet transforms) were developed in parallel: (i) a continuous wavelet transform (CWT) [34,40,41] and (ii) a discrete wavelet transform [36]. For singular (self-similar or multifractal) signals or images, the CWT transform rapidly became a predilection mathematical microscope to perform space-scale analysis and to characterize scale invariance properties $[37,38,43]$. In particular, it was used to elaborate a statistical physics formalism of multifractals [47 51]. During the past 30 years, the CWT was used for biological applications, on both one-dimensional (1D) signals and two-dimensional (2D) images [43 45,52 54]. 1D-CWT was applied to AFM force curves collected from single living plant cells [55], living mammalian cells [11 13 ] and to AFM fluctuation signals to characterize the passive microrheology of living myoblasts [31]. It was generalized to 2D [and to threedimensional (3D)] CWT [43,52,56] and it proved again its versatility and power for analyzing AFM topographic images of biosensors [57], fluorescence microscopy images of chromosome territories [58], and diffraction phase microscopy of living cells $[59,60]$.

The originality of this work is to use a wavelet-based time-frequency decomposition method for analyzing the fractional rheologic fluctuations of living cells when probed with an AFM cantilever and to demonstrate that these fluctuations are self-similar and Gaussian in the $(5 \mathrm{~Hz} 150 \mathrm{~Hz})$ range [the complex compliance $J(f) \propto f^{\alpha}$ ]. It also establishes the fractional exponent $\alpha$ as a fractional hereditariness marker [61] distinguishing myoblasts with internal cytoskeleton tensions (prestressed adherent cells, showing stiffer and longer actin stress fibers) from those which have lost their tensed actin network. In Sec. II, we describe the experimental methods, the rheological models, and the time frequency analysis with complex analytic wavelet transforms of AFM cantilever fluctuations. In Sec. III, we compare the fractional hereditariness of the different types of muscle precursor cells and the range of frequencies where their compliance $J(f)$ scales as a power law $\sim f^{\alpha}$. We conclude in Sec. IV.

\section{METHODS}

\section{A. Materials and methods}

\section{Cell culture and differentiation}

a. C2C12 myoblast culture. C2C12 myoblasts (ATCC CRL-1772) were cultured in a growth medium (GM) composed of high glucose (4.5 g/l) Dulbecco's Modified Eagle Medium (DMEM) with L-glutamine (PAA, GE Healthcare) supplemented with $20 \%$ fetal bovine serum (FBS, PAA), $1 \%$ penicillin streptomycin antibiotics $(100 \mathrm{U} / \mathrm{ml}$ penicillin and $100 \mu \mathrm{g} / \mathrm{ml}$ streptomycin, Gibco, Thermo Fisher Scientific) and $10 \mathrm{mM}$ HEPES (Gibco). GM was stored at $4{ }^{\circ} \mathrm{C}$ up to 1 month; fresh GM was replaced every 2 days. The myoblasts were maintained in a $5 \% \mathrm{CO}_{2}$ atmosphere at $37{ }^{\circ} \mathrm{C}$ inside 90 mm diameter Petri dishes until $\sim 70 \%$ confluency. To avoid that $6070 \%$ confluent myoblasts differentiate spontaneously, the dishes were washed with preheated PBS and cells were detached from the dish bottom with $0.25 \%$ trypsin EDTA (Gibco) for $3 \mathrm{~min}$ at $37^{\circ} \mathrm{C}$. Then, the cells were harvested 
and either replated at lower concentration or frozen-stored. Prior to AFM FIC collection, C2C12 myoblasts $\left(\sim 1.5 \times 10^{5}\right.$ cells from passages 10 to 14 ) were seeded on collagen coated Petri dishes, CCPDs (35 mm diameter) in GM and kept at least $24 \mathrm{~h}$ at $37^{\circ} \mathrm{C}$ with $5 \% \mathrm{CO}_{2}$. Then, the medium was changed and replaced by $2 \mathrm{ml}$ of GM and the Petri dish was transferred to the AFM. Each sample was used within $23 \mathrm{~h}$ and discarded afterwards.

b. C2C12 myoblast differentiation. Confluent (60\%) C2C12 myoblasts on CCPDs were induced to differentiation, replacing GM with a differentiation medium (DM) [62]. DM was composed of high glucose $(4.5 \mathrm{~g} / \mathrm{l})$ DMEM with L-glutamine (PAA), supplemented with $2 \%$ Donor Horse Serum (PAA), $1 \%$ penicillin streptomycin antibiotics (100 U/ml penicillin and $100 \mu \mathrm{g} / \mathrm{ml}$ streptomycin, Gibco), and $10 \mathrm{mM}$ HEPES (Gibco). The myoblasts were maintained in $\mathrm{DM}$ at $37^{\circ} \mathrm{C}$ with $5 \% \mathrm{CO}_{2}$ for at least 5 days, renewing the medium every 2 days. Different culture supports were used (glass bottom Petri dishes, SPDs, and CCPDs) to compare their impact on $\mathrm{C} 2 \mathrm{C} 12$ cell myogenic differentiation. C2C12 myoblast differentiation was monitored by DIC and time-lapse video recording. Multinucleated myotubes were observed with fluorescence confocal microscopy and probed by AFM at their 5th day of differentiation. A fusion index, defined as the percentage of nuclei contained in myotubes compared to the total number of nuclei observed in each image field, was used to quantify the level of myotube formation in different culture conditions.

C2C12 myoblasts grown on CCPDs (35 mm diameter) were rinsed twice with preheated PBS and fixed with freshly made $4 \%$ paraformaldehyde (PFA, Fluka, St. Louis, MO) in PBS for $20 \mathrm{~min}$ at room temperature (RT) $\left(24^{\circ} \mathrm{C}\right)$. Then, the sample was maintained in $2 \mathrm{ml}$ PBS before AFM measurements which lasted $\sim 4 \mathrm{~h}$ at RT.

c. Primary human myoblast culture. Purified primary human myoblasts from a patient (3 years old) affected by the DMD (V024) were courteously donated from Hospices Civils de Lyon (Centre de Biotechnologie Cellulaire, Groupement Hospitalier Est, Bron, France) after patient informed consent in accordance with the Declaration of Helsinki. The DMD patient carried deletions of axons 4852 in the dystrophin gene. The myoblasts were grown in proliferation medium (PM) at $37^{\circ} \mathrm{C}$ with $5 \% \mathrm{CO}_{2}$ on CCPDs. PM was composed of high glucose (4.5 g/l) DMEM with L-glutamine (PAA), supplemented with $20 \%$ FBS (PAA), $1 \%$ penicillin streptomycin antibiotics $(100 \mathrm{U} / \mathrm{ml}$ penicillin and $100 \mu \mathrm{g} / \mathrm{ml}$ streptomycin, Gibco), insulin $(10 \mu \mathrm{g} / \mathrm{ml}$, Sigma), human basic fibroblast growth factor (hbFGF, $25 \mathrm{ng} / \mathrm{ml}$, Sigma), and epidermal growth factor (EGF, $10 \mathrm{ng} / \mathrm{ml}$, Sigma). The filtered PM was stored at $4{ }^{\circ} \mathrm{C}$ up to 1 month; fresh PM was replaced every 2 days. DMD myoblasts were dissociated from the culture substrates either for routine subculture or for cryopreservation using $0.05 \%$ trypsin EDTA (Gibco) for $35 \mathrm{~min}$ at $37^{\circ} \mathrm{C}$ after replacement of PM with preheated PBS.

DMD primary human myoblasts were seeded $\left(2.5 \times 10^{4}\right.$ cells from 2 nd and $3 \mathrm{rd}$ passages) on CCPDs (35 $\mathrm{mm}$ diameter) in $\mathrm{PM}$ and kept at $37^{\circ} \mathrm{C}$ with $5 \% \mathrm{CO}_{2}$ at least $24 \mathrm{~h}$ before AFM recording. Then, the medium was changed and replaced by $2 \mathrm{ml}$ of $\mathrm{PM}$ and the Petri dish was transferred to the AFM. Each sample was used within $23 \mathrm{~h}$ and discarded afterwards.

d. ATP depletion. Living cells rely on a combination of oxidative and glycolytic energy metabolism for ATP production. For a complete ATP depletion, both pathways must be inhibited [63], namely, the mitochondrial electron transport chain (ETC) complex III with antimycin A (AMA) [64] and glycolysis with 2-deoxy-D-glucose (2-DG) [65]. ATP depletion buffer composition: $140 \mathrm{mM} \mathrm{NaCl}, 5 \mathrm{mM} \mathrm{KCl}, 1 \mathrm{mM}$ $\mathrm{MgCl}_{2}, 2 \mathrm{mM} \mathrm{CaCl}$, $10 \mathrm{mM}$ HEPES, $6 \mathrm{mM}$ 2-DG (Sigma), and $5 \mu \mathrm{M}$ AMA (Sigma). The filtered solution was adjusted to $p \mathrm{H}=7.4$ and stored at $-20^{\circ} \mathrm{C}$. CCPDs with adherent C2C12 myoblasts were first rinsed twice with preheated PBS and filled with freshly thawed ATP depletion buffer ( $2 \mathrm{ml}$ ). Fifteen minutes was necessary for the cellular ATP concentration to decrease below $7 \%$ of its initial concentration [66]. AFM FIC capture was performed on single cells, inside the ATP-depletion buffer at RT for $23 \mathrm{~h}$, before discarding the CCPD.

e. Blebbistatin treatment. $\mathrm{C} 2 \mathrm{C} 12$ myoblasts were treated with (S)-(-)-Blebbistatin (Santa Cruz Biotechnology) to inhibit the activity of the nonmuscle myosin II [67]. Aliquots of $100 \mu \mathrm{M}$ blebbistatin dissolved in dimethylsulfoxide (DMSO) were stored at $-20{ }^{\circ} \mathrm{C}$. GM was replaced with the same amount $(2 \mathrm{ml})$ of preheated GM containing blebbistatin $(50 \mu \mathrm{M})$ for 20 min [68]. AFM experiments on C2C12 cells inside GM-blebbistatin were performed in $\sim 2.5 \mathrm{~h}$ at RT.

f. Petri dish surface treatment. We tested different surface treatments: gold coating, standard (SPD), and type I collagen coated petri dishes (CCPDs), and we observed that CCPDs lead to greater $\mathrm{C} 2 \mathrm{C} 12$ myoblast lengths, widths, and areas. In this study, we chose type I collagen for myoblast adhesion because this protein is one of the major insoluble fibrous protein found in the in vivo extracellular matrix. Type I collagen aqueous solution $(3 \mathrm{mg} / \mathrm{ml})$ from bovine skin and tendon BioReagent (Sigma-Aldrich) was diluted in ultra-pure water to get $100 \mu \mathrm{g} / \mathrm{ml}$. Petri dishes were incubated $3 \mathrm{~h}$ with this solution $\left(8 \mu \mathrm{g} / \mathrm{cm}^{2}\right)$ at $37^{\circ} \mathrm{C}$ to allow proteins to bind, dried overnight at RT under clean atmosphere, and rinsed with Dulbecco's Phosphate Buffer Saline (PBS, Sigma) before use.

\section{Fluorescence microscopy and staining}

Fluorescence confocal microscopy imaging was performed with an inverted scanning confocal microscope (ZEISS LSM710) equipped with a motorized X Y stage and a set of ZEISS objectives with magnification from $10 \times$ to $63 \times$. Fluorescent images $(1224 \times 900$ pixels $)$ were collected using a $405 \mathrm{~nm}$ blue-violet laser diode (DAPI, AMCA), a $488 \mathrm{~nm}$ argon laser (Alexa Fluor 488), and a $561 \mathrm{~nm}$ diodepumped solid state (DPSS) laser (Cy3). The excitation and the fluorescence signal acquisition of each fluorophore were executed in sequential multitracking channels in order to avoid bleed-through crossover artifacts. Saturation was checked and avoided using range indicators of the Zen software. The scan speed and image averaging were optimized before image acquisition in order to have the highest 

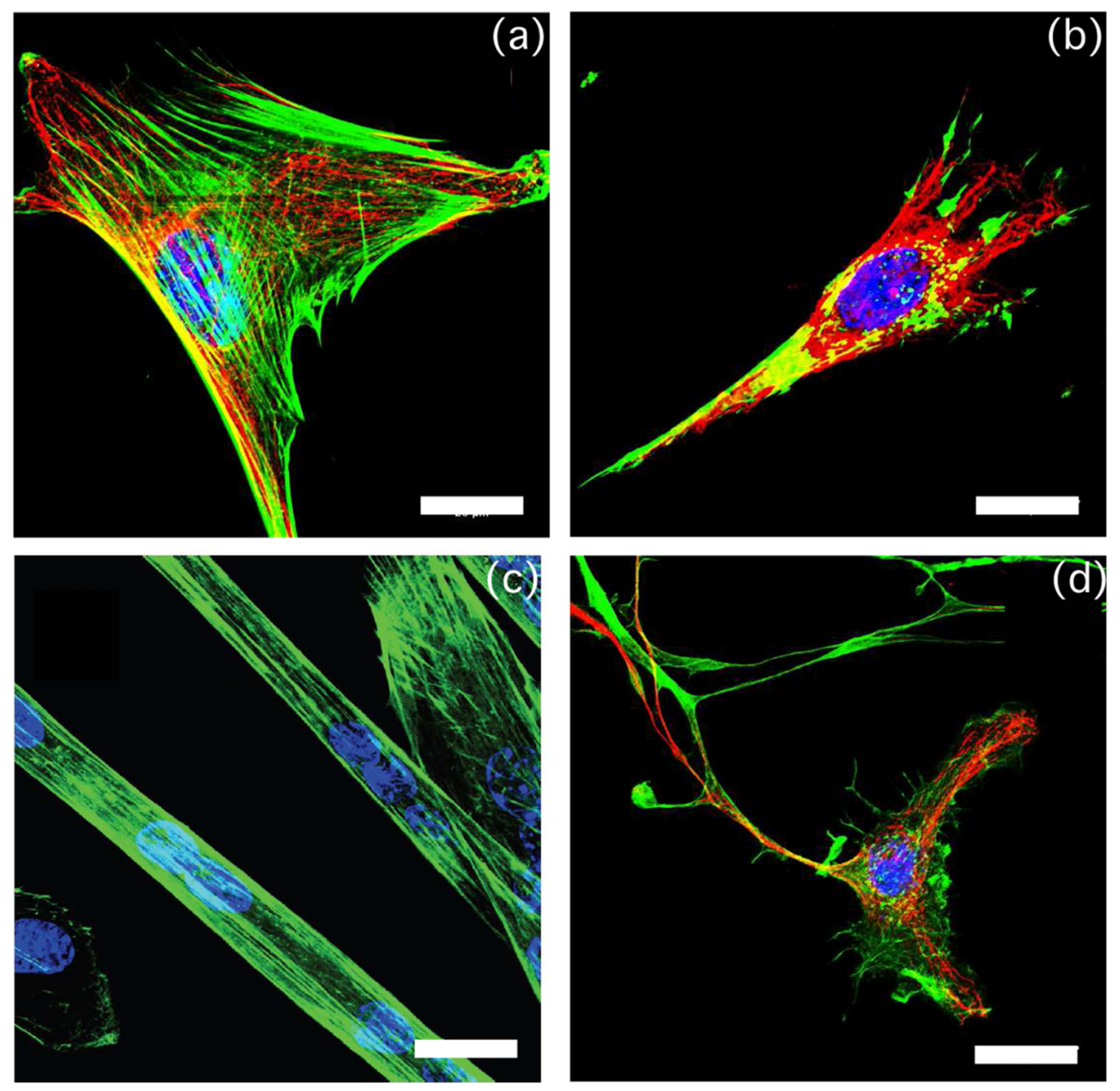

FIG. 1. Fluorescence images of $\mathrm{C} 2 \mathrm{C} 12$ myoblast cells $[(\mathrm{a}),(\mathrm{b})$, and (d)] and $\mathrm{C} 2 \mathrm{C} 12$ myotubes (c) showing their nuclei (DAPI blue), actin filaments (phalloi din Alexa Fluor 488 green), and microtubules ( $\beta$ tubulin Cy3 conjugate red). (a) Normal myoblast cell. (b) Myoblast cell treated with an ATP depletion buffer. (c) Normal myotubes after 5 days of differentiation. (d) Myoblast cells treated with a blebbistatin buffer. Scale bar: $20 \mu \mathrm{m}$. A color Fig. 1 is found in the online version of the manuscript.

signal-to-noise ratio for a minimum cost of time. Z-Stack images of $9 \mu \mathrm{m} \pm 3 \mu \mathrm{m}$ depth with $0.5 \mu \mathrm{m}$ interval between each slice were acquired with the Zen software. ImageJ $1.47 \mathrm{v}$ processing program was used to analyze the confocal microscopy images and to reconstruct the $\mathrm{Z}$ projections.

Fluorescent labelling of specific intracellular structure of $\mathrm{C} 2 \mathrm{C} 12$ cells was performed combining immunofluorescence and nonantibody labelling techniques. Adherent C2C12 myoblasts $\left(\sim 10^{5}\right)$ and confluent myotubes on $35 \mathrm{~mm}$ diameter CCPDs were rinsed twice with preheated PBS and fixed with freshly made 4\% PFA (Fluka) in PBS for $20 \mathrm{~min}$ at room temperature (RT). The samples were then permeabilized with 0.05\% Triton X-100 (Euromedex, Souffelweyersheim, France) in PBS for 10 min at RT before being saturated with $1 \%$ bovine serum albumin (BSA, Sigma) in PBS and kept for $30 \mathrm{~min}$ at RT. The samples were stained either for F-actin, $\beta$-tubulin, and nucleus or for the three cytoskeleton filaments according to the two following protocols. In the first case, after incubation with mouse monoclonal Cy3-conjugate anti- $\beta$-tubulin (Sigma) diluted 1:200 in 1\% BSA PBS for $1 \mathrm{~h}$ at RT in a humid and dark chamber, the cells were incubated with phalloidin-Alexa Fluor 488 (Molecular Probes) diluted 1:100 in 1\% BSA PBS for 15 min at RT in a humid and dark chamber. Finally, the samples were sealed with glass cover-slides and VectaShield mounting medium (Vector Laboratories) with DAPI $\left(4^{\prime}, 6\right.$-diamino-2- phenylindole) and stored at least for $10 \mathrm{~min}$ at $4{ }^{\circ} \mathrm{C}$. In the second case before $\beta$-tubulin staining, the cells were incubated with mouse monoclonal anti-desmin (DAKO, Agilent Technologies) diluted 1:200 in 1\% BSA PBS over night in a humid chamber at $4{ }^{\circ} \mathrm{C}$. The day after, incubation with AMCA-conjugated goat secondary antibody anti mouse (Jackson ImmunoResearch) diluted 1:1000 in 1\% BSA PBS was carried out for $1 \mathrm{~h}$ at RT in a humid and dark chamber. Then, the labelling of $\beta$-tubulin and F-actin was performed similarly to the previous protocol before sealing the samples with glass cover-slides and VectaShield mounting medium without DAPI and storing them at least $10 \mathrm{~min}$ at $4{ }^{\circ} \mathrm{C}$. In both protocols, the samples were gently rinsed with PBS three times for $5 \mathrm{~min}$ between each step. Fluorescence images of $\mathrm{C} 2 \mathrm{C} 12$ myoblasts and myotubes in different conditions are shown in Fig. 1.

\section{AFM experimental protocols and cantilever calibration}

A CellHesion 200 Atomic Force Microscope (AFM, JPK Instruments) coupled to a transmission inverted microscope 
and a CCD camera was used for nano-indentation experiments. The apparatus was equipped with an $X Y$ Motor Precision Stage (JPK) with $20 \times 20 \mathrm{~mm}$ motorized stage, a vibration isolation table (Melles Griot), a foam-based acoustic isolation system, and a white light LED illumination (Thorlabs, MCWHLS). The AFM Z-piezotransducer with movement range of $100 \mu \mathrm{m}$ was controlled by a closed loop feedback system with subnanometric precision. Proportional gain ( $P$ gain) was set at 20 and integral gain ( $I$ gain) at 0.002 .

Creep (fixed force) experiments were performed with two types of AFM probes: (i) triangular gold-coated silicon nitride cantilevers from Sharp Nitride Lever (SNL-10, Bruker, Camarillo, CA) with nominal spring constant of $0.06 \mathrm{~N} / \mathrm{m}(\min =0.03 \mathrm{~N} / \mathrm{m} ; \max =0.12 \mathrm{~N} / \mathrm{m})$ and typical resonant frequency in air: $18 \pm 6 \mathrm{kHz}$; (ii) rectangular partially gold-coated quartz cantilevers (qp-CONT 20, Nanosensors, Neuchatel, Switzerland) with nominal spring constant of $0.1 \mathrm{~N} / \mathrm{m}(\min =0.08 \mathrm{~N} / \mathrm{m} ; \max =0.15 \mathrm{~N} / \mathrm{m})$ and typical resonant frequency in air: $30 \pm 4 \mathrm{kHz}$. Before each experiment, cantilever calibration was carefully performed both in air, to verify the correct positioning of the probe and the proper system alignment, and in liquid to estimate the cantilever spring constant $(k)$. First, the deflection sensitivity $(\mathrm{nm} / \mathrm{V})$ of the cantilever-photodiode system was evaluated using the in-contact part of force indentation curves (FICs) on a clean glass surface (five FICs collected in both air and liquid).

a. AFM cantilever calibration. The cantilever spring constant was calibrated in both air and liquid by the thermal noise method $[69,70]$. From the thermal fluctuations of the cantilever, its spring constant $k$ can be estimated, assuming harmonicity (small amplitude fluctuations) and energy equipartition [69]: $k=k_{B} T /\left\langle d^{2}\right\rangle$, where $\left\langle d^{2}\right\rangle$ is the mean square displacement of the cantilever from its neutral position, $T$ the absolute temperature in Kelvin, and $k_{B}$ the Boltzmann's constant. Since AFM cantilevers have different geometries and several vibration modes, a correction factor $\kappa$ must be included: $k=\kappa k_{B} T /\left\langle d^{2}\right\rangle$, where the factor $\kappa$ varies according to the vibration mode and the cantilever geometry [71]. The value $\kappa=0.817$ (respectively 0.778 ) was used for rectangular (respectively triangular) cantilevers.

Power spectral density was computed from AFM cantilever deflection signals captured $100 \mu \mathrm{m}$ away from the sample surface. Finally, the vertical deflection $(\Delta D)(\mathrm{nm})$ of the cantilever being proportional to the force applied to the sample, we converted it to a tip sample interaction force $\Delta F(\mathrm{nN})$ knowing the stiffness of the cantilever $k(\mathrm{~N} / \mathrm{m})$ through Hooke's law: $\Delta F=k \Delta D$ and the sensitivity of the photodiode quadrant.

b. Creep (constant force) experiment protocol. AFM constant force experiments were performed with a CellHesion 200 Atomic Force Microscope (JPK Instruments, Berlin, Germany) coupled to a transmission inverted microscope, a white light LED illumination (Thorlabs, Model MCWHLS, Newton, NJ), a CCD camera (The Imaging Source Europe, Bremen, Germany), a $20 \times 20 \mathrm{~mm}$ X-Y Motor Precision Stage (JPK), a vibration isolation table (Melles Griot, Albuquerque, NM), and a foam-based acoustic isolation system. We used qp-Cont-20 rectangular quartz-like cantilevers (Nanosensors TM, Neuchatel, Switzerland), which have the following specifications: thickness $0.75 \pm 0.03 \mu \mathrm{m}$, length $125 \pm 5 \mu \mathrm{m}$, width $35 \pm 2 \mu \mathrm{m}$, resonance frequency $2634 \mathrm{kHz}$, nominal force constant $0.1 \mathrm{~N} / \mathrm{m}(\min =0.08 \mathrm{~N} / \mathrm{m}$; $\max =0.15 \mathrm{~N} / \mathrm{m}$ ), and tip height: $68 \mu \mathrm{m}$. Before recording the cantilever fluctuation signals, the system alignment and the thermal noise cantilever calibration were carried out on a $35 \mathrm{~mm}$ Petri dish with a glass bottom filled with $2 \mathrm{ml}$ of ultra-pure water. A thermal noise signal (100 s) was first recorded in these conditions at $100 \mu \mathrm{m}$ above the dish surface. Then, the dish with water was discarded and replaced with a collagen coated $35 \mathrm{~mm}$ Petri dish containing the living cells $\left(\sim 1.5 \times 10^{5}\right.$ cells from passages 10 to 14$)$ in $2 \mathrm{ml}$ of culture medium. The chamber thermal noise signal was again captured at $100 \mu \mathrm{m}$ above the dish surface on a region close to the probed cell $(\leq 50 \mu \mathrm{m})$, then the cantilever tip was approached to the cell nuclear region, using the JPK constant force mode (force set point $=1 \mathrm{nN}$, proportional gain $=20$, integral gain $=0.002$ ) and maintained at this force of $1 \mathrm{nN}$ for the cantilever fluctuations recording. After a time delay of $5 \mathrm{~s}$, required for the cantilever approach to the cell and cantilever contact force establishment, the cantilever height, error, lateral and vertical deflection signals were recorded during $100 \mathrm{~s}$ with an acquisition rate of $800 \mathrm{kHz}$.

\section{B. Theory/Calculation}

\section{Formalism for the fractional rheology of cells}

Fractional calculus provides a natural framework for describing the power-law rheology of living cells [72]. Here, we adapt this formalism to reformulate the hereditary integrals which have been proposed for modelling stress relaxation or creep experiments with spherical or conical indenters [73 75].

General linear constitutive equations for viscoelastic materials were formulated from hereditary (convolution) integrals [73]. They were further extended to pyramidal or conical indentations [74,75]. For creep experiments (fixed loading force $F$ ) with conical shape indenters, the square of the depth of indentation $d$ reads as a convolution integral of the force derivative:

$$
d^{2}(t)=\mathcal{D} \int_{0}^{t} J(t-\tau) d F(\tau)
$$

where $J$ is the creep compliance, $D=\frac{\pi(1 v)}{4 \tan \theta}, v$ is the Poisson coefficient, and $\theta$ the nominal tip half-angle. We assume in this study that the cells behave as isotropic viscoelastic materials and we take $v=0.5$ as a first approximation. Actually, living cells are not isotropic, and their Poisson coefficient may not be invariant in time. A first attempt to generalize hereditary integrals to anisotropic materials with fractional rheology was recently published [76], and could be extended for modelling the indentation of anisotropic living cells with axisymmetric indenters of variable shape.

The principle of a creep experiment is formalized by the following equation for the force: for $t>0: F_{m}(t)=F_{0} H(t)$, where $H(t)$ is the Heaviside step function. To reach and maintain this mean loading force, the vertical position of the 
AFM cantilever is adjusted in real time. In real experiments, around this mean loading force $F_{m}$, the fluctuations of $F$ are the consequence of either thermal noise-driven fluctuations or mechanical vibrations of the experiment:

$$
F(t)=F_{m}(t)+S_{\mathrm{F}}(t)=F_{0} H(t)+S_{\mathrm{F}}(t) .
$$

If we assume that the fluctuations $S_{\mathrm{F}}(t)$ are stochastic and uncorrelated, they can be modeled by a zero mean Gaussian stochastic process for which a flat power spectrum density has been defined.

As the distributional derivative of the Heaviside step function is the Dirac delta distribution: $d H(t)=\delta(t) d t$, Eq. (1) can be rewritten as

$$
d^{2}(t)=\mathcal{D}\left[F_{0} J(t)+\int_{0}^{t} J(t-\tau) d S_{\mathrm{F}}(\tau)\right] .
$$

$d^{2}$ can be expressed as the sum of two terms:

$$
\begin{aligned}
d^{2}(t) & =\left[d_{m}(t)+S_{d}(t)\right]^{2} \\
& =d_{m}^{2}(t)+2 d_{m}(t) S_{d}(t)+\left[S_{d}(t)\right]^{2},
\end{aligned}
$$

where $d_{m}^{2}(t)=D F_{0} J(t)$ is a mean time-varying value and $S_{d}(t)$ is a fluctuating term of much smaller amplitude. Given that $d_{m}(t)$ varies on much longer time scales than $S_{d}(t)$, for the range of frequencies investigated here $([1 \mathrm{~Hz}, 1 \mathrm{kHz}])$, the low frequency dynamics will be separated from the higher frequency fluctuations: $d_{m}(t) \sim d_{m}=$ Cste. Since moreover $S_{d}(t) \ll d_{m}$, the term of order 2 in $S_{d}(t)$ in Eq. (4) can be neglected and we get the expression for $S_{d}(t)$ :

$$
S_{d}(t)=\frac{\mathcal{D}}{2 d_{m}} \int_{0}^{t} J(t-\tau) d S_{\mathrm{F}}(\tau) .
$$

Structural damping materials are characterized by temporal power-law compliances $J(t)\left[\begin{array}{ll}77 & 80\end{array}\right]$ :

$$
J(t)=\frac{t^{\alpha}}{C_{\alpha} \Gamma(1+\alpha)},
$$

where $C_{\alpha}=E_{0}\left(\frac{C_{\eta}}{E_{0}}\right)^{\alpha}$ has a dimension that depends on $\alpha$, and $\Gamma$ is the Euler gamma function. $C_{\alpha}$ reduces to a Young modulus $E_{0}$ when $\alpha=0$ and to a viscosity coefficient $C_{\eta}$ when $\alpha=1$. For $0<\alpha<1$, the parameters $E_{0}$ and $C_{\eta}$ do not have these physical meanings. Thus, the integral of Eq. (5) transforms into a fractional integral of the force fluctuations:

$$
S_{d}(t)=\frac{\mathcal{D}}{2 d_{m} C_{\alpha} \Gamma(1+\alpha)} \int_{0}^{t}(t-\tau)^{\alpha} d S_{\mathrm{F}}(\tau) .
$$

A simple integration by part leads to

$$
\begin{aligned}
S_{d}(t) & =\frac{\mathcal{D}}{2 d_{m} C_{\alpha} \Gamma(\alpha)} \int_{0}^{t}(t-\tau)^{\alpha}{ }^{1} S_{\mathrm{F}}(\tau) d \tau \\
& =\frac{\mathcal{D}}{C_{\alpha}}\left(I_{0+}^{\alpha} S_{\mathrm{F}}\right)(t),
\end{aligned}
$$

where $\left(I_{0+}^{\alpha} S_{\mathrm{F}}\right)(t)$ denotes the Riemann-Liouville fractional integral of $S_{\mathrm{F}}$ [81]. Thus, $S_{d}(t)$ can be interpreted as the fractional integration of exponent $\alpha$ of the force fluctuations $S_{\mathrm{F}}$. Equation (8) can be rewritten in Fourier space as

$$
\widehat{S_{d}}(f)=\int_{\infty}^{\infty} S_{d}(t) e^{2 \pi i f t} d t=\frac{\mathcal{D}}{2 d_{m} C_{\alpha}}[i f]{ }^{\alpha} \widehat{S_{\mathrm{F}}}(f)
$$

Equation (9) is an infinite integral over a continuously varying time variable. In practice, discrete Fourier transforms are used in numerical computations and require some precautions. Before starting the experiments, the range of frequency for characterizing the rheology of cells has to be chosen. This frequency range is used to fix the temporal discretization (time step $\delta T$ ) and the total duration of the recorded signal. $\delta T$ was taken such that the largest frequency of the corresponding power spectrum $f_{s}=1 / \delta T$ be larger than the upper bound of the frequency domain (to avoid aliasing effects). Similarly, the duration of the signal was fixed to be much larger $(10 \times)$ than the inverse of the minimum frequency, to eliminate boundary effects for the wavelet transform computation.

If the sample compliance does not change during the experiment (avoiding active processes) and GSER [21,22] is valid, the complex compliance varies as a power law of the frequency $[f=\omega /(2 \pi)]$ :

$$
\widehat{J}(f)=\frac{\widehat{S_{d}}(f)}{\widehat{S_{\mathrm{F}}}(f)}=\frac{\mathcal{D}}{2 d_{m} C_{\alpha}}[i f]^{\alpha} .
$$

\section{Time-frequency analysis of noisy signals with the wavelet transform}

a. Wavelet transform mathematical formulation. The wavelet transform $T_{S_{d}}(b, a)$ of a signal $S_{d}(t)$ consists of its decomposition in terms of wavelets which are constructed from a chosen analyzing wavelet $\psi$ by means of translations (time parameter $b$ ) and dilations (scale parameter $a>0$ ). It is written as a convolution product of $S_{d}(t)$ with the analyzing wavelet $\psi$ shifted by $b$ and scaled by $a[40,41]$ :

$$
\begin{aligned}
T_{\psi}\left[S_{d}\right](b, a) & =\frac{1}{a^{1 / 2}} \int_{\infty}^{\infty} S_{d}(t) \psi^{*}\left[\frac{t-b}{a}\right] d t \\
& =a^{1 / 2} \int_{\infty}^{\infty} \widehat{S}_{d}(f) \hat{\psi}^{*}(a f) e^{2 \pi i f b} d f,
\end{aligned}
$$

where we have chosen to use the $\mathcal{L}^{2}$ norm that preserves the modulus square (energy) of the wavelet. We note $\hat{\psi}^{*}$ the complex conjugate of $\hat{\psi}$. The scale $a$ is a nondimensional quantity, it can be defined as an inverse of the frequency $a=f_{0} / f$. In this study, we took $f_{0}$ equal to the peak frequency $f_{\hat{\psi}^{\max }}$ (see supplementary material [103]).

Usually $\psi$ is only required to be of zero mean, but for the particular purpose of singularity detection of interest here, we will also require $\psi$ to be orthogonal to some low-order polynomials [i.e., to have $N$ vanishing moments $\int_{\mathbb{R}} x^{n} \psi(t) d t=0$, $n=1,2, \ldots, N-1$ ] [82]. For the wavelet $\psi$ to be admissible, its Fourier transform must fulfill the condition:

$$
C_{\psi}=\int_{\infty}^{\infty} \frac{|\hat{\psi}(f)|^{2}}{|f|} d f<\infty
$$



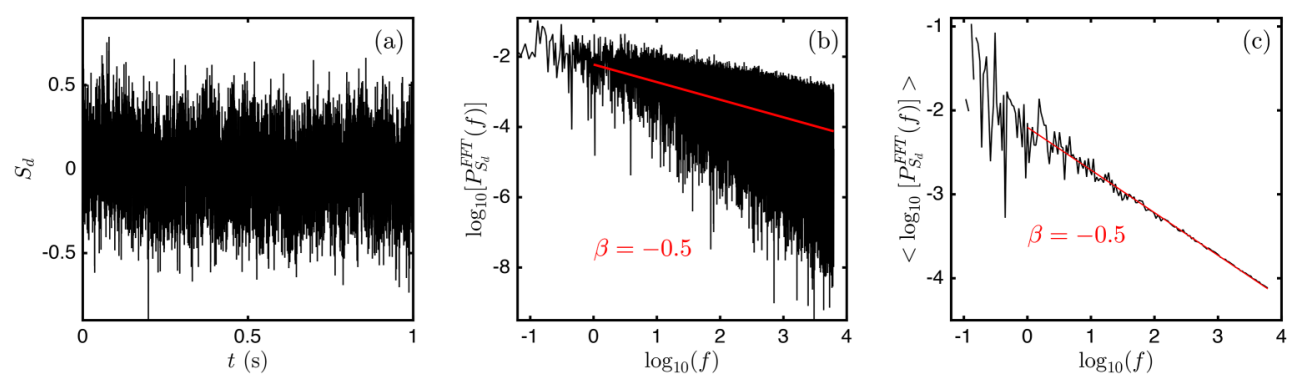

FIG. 2. Power spectrum of a fractional Gaussian stochastic signal computed with a standard FFT transform. (a) The signal $S_{d}(t)$ (a $1 \mathrm{~s}$ portion of this numerical signal of length $80 \mathrm{~s}$ ) with Hölder exponent $h \quad 0.25$. (b) FFT power spectrum. (c) Averaged FFT power spectrum of $S_{d}$ on logarithmically distributed fre quency values. The total length and sampling time of this numerical signal are identical to the characteristics of the experimental AFM signals investigated in this work.

This time frequency analytic tool has proved particularly efficient for the analysis of complex nonstationary signals, and signals involving a mixture of periodic and stochastic components. In particular, continuous wavelets constructed from derivatives of Gaussian functions were used to track local singularities [83 89] and a whole formalism for the statistical thermodynamics of fractals was elaborated [47 51,90,91]. For the detection of rhythms (periodic or quasiperiodic components) from noisy signals, complex-valued square integrable wavelets, $g_{c s i}$ wavelets, were proposed such that their Fourier transform vanishes for negative frequency values (analytic functions) [92 94]. Analytic wavelet transforms provide both the magnitude and the phase information of a signal in the time frequency domain.

$b$. Morse wavelets. The generalized Morse wavelets constitute a two-parameter family of exactly analytic continuous wavelets. They have been introduced in the late 1980s to define time frequency localization operators $[95,96]$ and revisited more recently for their application to complex (noisy) signals [97 100]. These wavelets are defined in the frequency domain $(\omega=2 \pi f)$ as

$$
\begin{aligned}
& \widehat{\psi}_{n, \gamma}(\omega)=C_{n, \gamma} \omega^{n} e^{\omega^{\gamma}}, \quad \text { for } \omega \geq 0, \\
& \widehat{\psi}_{n, \gamma}(\omega)=0, \quad \text { for } \omega<0,
\end{aligned}
$$

where $C_{n, \gamma}$ is a normalization constant, $n$ and $\gamma$ are two parameters controlling the wavelet shape. Equation (13) confers the analyticity to the generalized Morse wavelets. The maxima of $\widehat{\psi}_{n, \gamma}(\omega)$ (the peak frequency) corresponds to frequencies such that $n-\gamma \omega^{\gamma}=0$, that is, $\omega_{0}=2 \pi f_{0}=$ $(n / \gamma)^{\frac{1}{\gamma}}$ [see the supplementary material [103] for the expression of the derivative of $\left.\widehat{\psi}_{n, \gamma}(\omega)\right]$. The Morse wavelet family includes as special cases the Cauchy wavelets $\widehat{\psi}_{n, 1}(\omega)$ $(\gamma=1)$, as well as analytic versions of the derivative of Gaussian wavelets $\widehat{\psi}_{n, 2}(\omega)(\gamma=2)$, and also the Airy wavelets $(\gamma=3)$.

We illustrate in Fig. S1 [103] Morse wavelets for different values of $\gamma$ and $n$. The solid (respectively dashed) lines correspond to $\gamma=1$ (respectively $\gamma=2$ ). Increasing $n$ confers an increasing number of oscillations to the wavelet, and a peak frequency increase in the Fourier domain. The shape of the wavelet varies also with $\gamma$, the larger $\gamma$, the sharper and the smaller the wavelet maxima frequency, and the greater its number of oscillations. To detect local frequencies in a signal, the greater $n$ and $\gamma$ values will be the more efficient. On the contrary to extract power-law behaviors which occur on wider frequency ranges, smaller $n$ and $\gamma$ values will be preferred. In this perspective, we chose here a Cauchy wavelet $(\gamma=1)$ with $n=1$ (Fig. S1 [103]).

c. Time-frequency decomposition of the energy of a signal. The total energy of a signal $S_{d}(t)$ is given by

$$
I_{S_{d}}=\int_{\infty}^{\infty}\left|S_{d}(t)\right|^{2} d t=\int_{\infty}^{\infty}\left|\widehat{S}_{d}(f)\right|^{2} d f .
$$

This total energy of $S_{d}$ can be recovered from its wavelet decomposition. Let us define the integral of the square modulus of the wavelet transform of $S_{d}$ :

$$
I_{T_{\psi}\left[S_{d}\right]}=\iint_{\mathbb{R}_{+}^{*} \times \mathbb{R}}\left|T_{\psi}\left[S_{d}\right](b, a)\right|^{2} \frac{d a}{a^{2}} d b .
$$

Thanks to Parseval theorem, Eq. (11) and the change of notation $\Omega=a f$, with $a>0 \quad(d a / a=d \Omega / \Omega)$, we can rewrite Eq. (15) in the following form as

$$
I_{T_{\psi}\left[S_{d}\right]}=\iint_{\mathbb{R}_{+}^{*} \times \mathbb{R}_{+}^{*}}\left|\widehat{S}_{d}(f)\right|^{2}|\hat{\psi}(\Omega)|^{2} \frac{d \Omega}{\Omega} d f .
$$

Note that the range of integration of $f$ and $\Omega$ has been limited to positive values because we have chosen an analytic analyzing wavelet (null for negative $\Omega$ values). The total energy computed from the wavelet transform of $S_{d}$ is, therefore, proportional to its total energy:

$$
I_{T_{\psi}\left[S_{d}\right]}=C_{\psi} I_{S_{d}}=C_{\psi} \int_{\infty}^{\infty}\left|\widehat{S}_{d}(f)\right|^{2} d f
$$

where we recognize the admissibility constant $C_{\psi}$ [Eq. (12)]. Equation (17) can be rewritten as

$$
\begin{aligned}
I_{T_{\psi}\left[S_{d}\right]} & =C_{\psi} \int_{\infty}^{+\infty} P_{S_{d}}^{\mathrm{FFT}}(f) d f, \\
& =C_{\psi} \int_{\infty}^{+\infty} P_{S_{d}}^{\mathrm{CWT}}(f) d f,
\end{aligned}
$$

where $P_{S_{d}}^{\mathrm{FFT}}$ is the power spectrum of $S_{d}$ usually obtained 

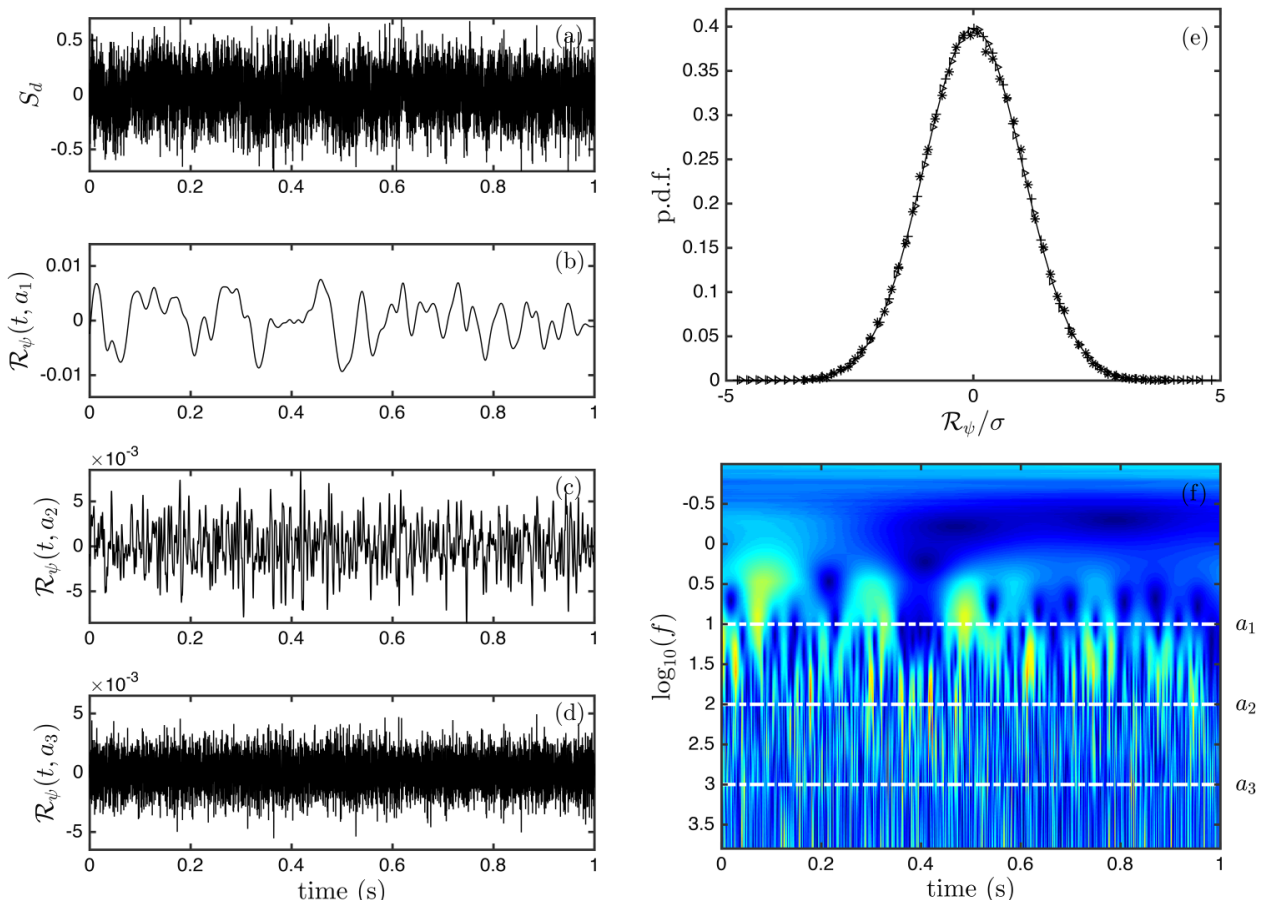

FIG. 3. Wavelet based spectral decomposition of a synthetic fractional Gaussian random signal $S_{d}$ with Hölder exponent $h \quad 0.25$, with a Morse wavelet of

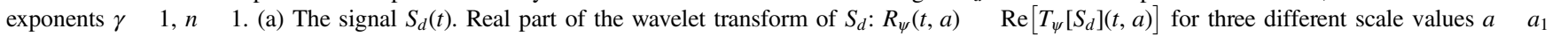
$f_{0} / 10$ (b), $a \quad a_{2} \quad f_{0} / 100$ (c), and $a \quad a_{3} \quad f_{0} / 1000$ (d). (e) P.d.fs of $R_{\psi}(., a) / \sigma(a)$ computed at scales $a_{1}$ (star), $a_{2}$ (cross), and $a_{3}$ (triangle) after rescaling by the corresponding mean standard deviation $\sigma(a)$. (f) Color coded representation of the modulus of $T_{\psi}\left[S_{d}\right](t, a)$. Each line has been coded separately from 0 (dark blue) to 1 (red).

from classical Fourier analysis, and

$$
P_{S_{d}}^{\mathrm{CWT}}(f)=\frac{1}{f_{0} C_{\psi}}\left[\int_{\infty}^{+\infty}\left|T_{\psi}\left[S_{d}\right]\left(b, \frac{f_{0}}{f}\right)\right|^{2} d b\right]
$$

is the wavelet-based power spectrum of the signal $S_{d}$.
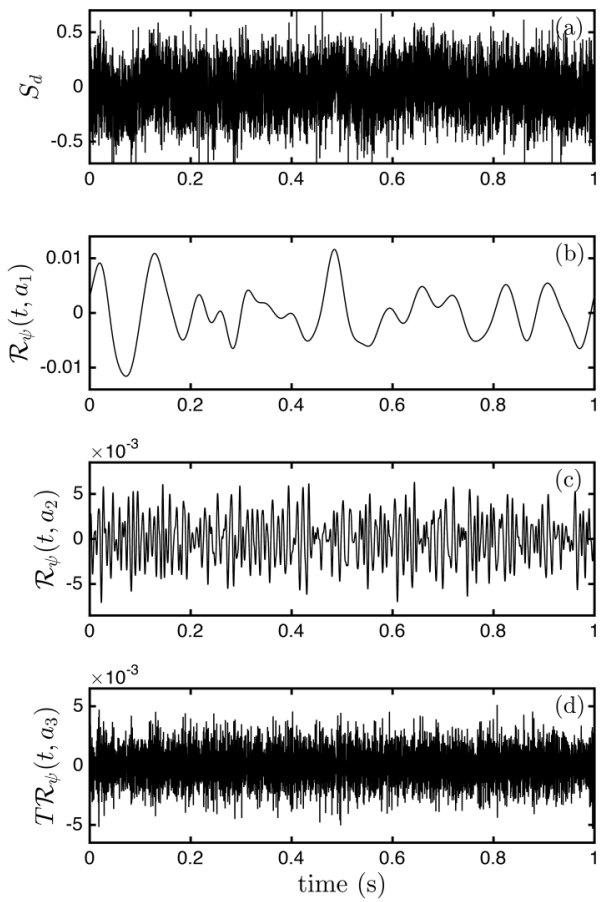

\section{Wavelet-based power spectra of synthetic signals}

We use fractional zero mean Gaussian random signals to illustrate the performance of wavelet-based power spectra, as compared to standard FFT computation. Such signals are
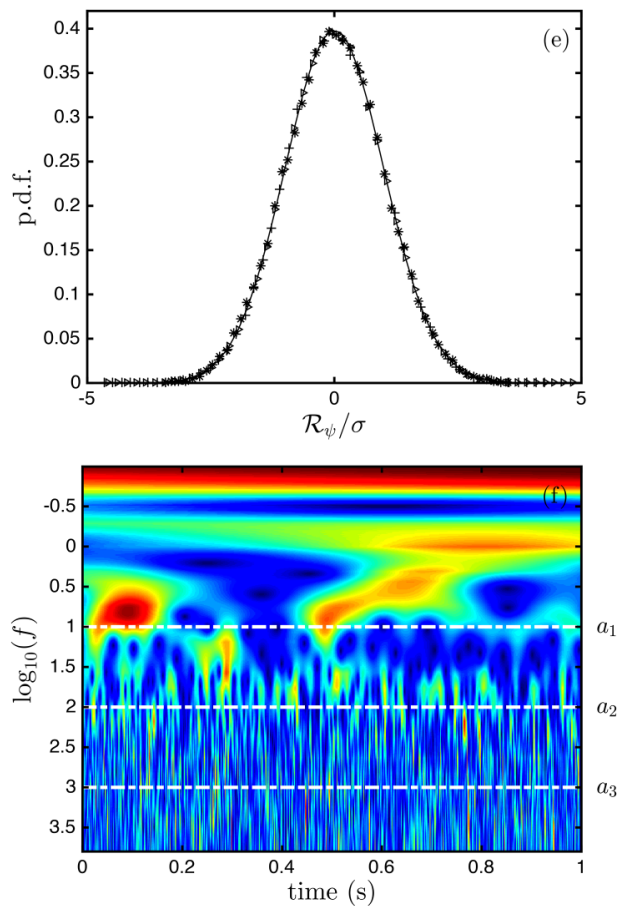

FIG. 4. Wavelet based spectral decomposition of a synthetic fractional Gaussian random signal $S_{d}$ with Hölder exponent $h$ exponents $\gamma \quad 1, n \quad$ 4. Same representation as in Fig. 3. 

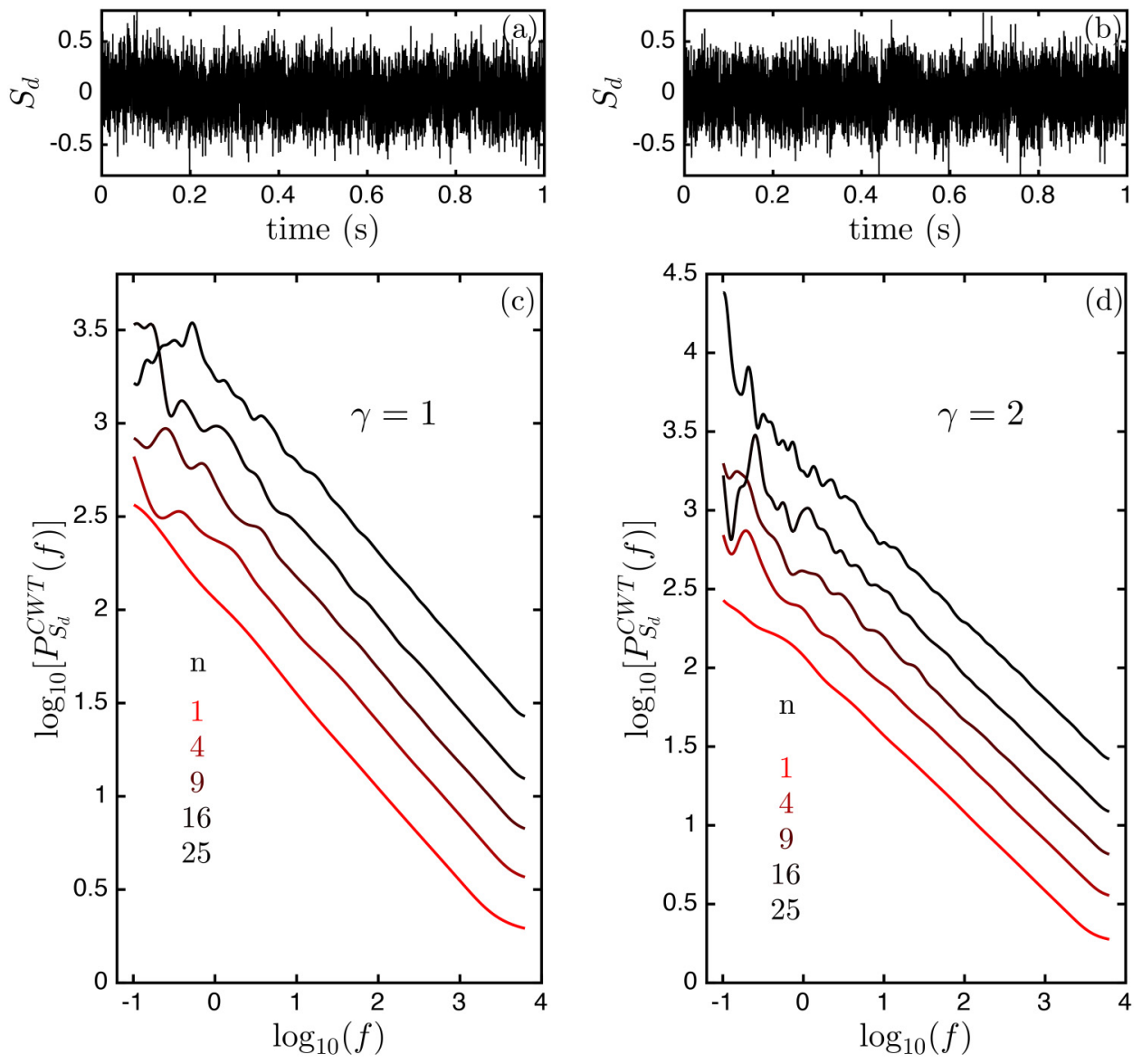

FIG. 5. Comparison of the wavelet based power spectra of fractional Gaussian random signals with Hölder exponent $h \quad 0.25$, computed with Morse wave lets for different $n$ values. [(a) and (b)] The signal $S_{d}(t)$. [(c) and (d)] The power spectra $\log _{10} P_{S_{d}}^{C W T}(f)$ vs $\log _{10}(f)$ computed with a Morse wavelet of exponents $\gamma \quad 1$ (left) and $\gamma \quad 2$ (right) with different $n$ values $(n \quad 1,4,9,16,25)$. The power spectra were shifted vertically for a better comparison.

characterized by a noninteger power-law scaling of their Fourier transform: $\widehat{S}_{d}(f) \propto f^{\alpha}$. Their wavelet transform with a Morse analyzing wavelet of parameters $n$ and $\gamma$ reads

$$
T_{\psi_{n, \gamma}}\left[S_{d}\right](b, a) \propto a^{1 / 2} \int_{\infty}^{+\infty} f^{\alpha} \widehat{\psi}_{n, \gamma}^{*}(a f) e^{2 i \pi f b} d f .
$$

Replacing $\widehat{\psi}_{n, \gamma}^{*}(f)$ by its expression [Eq. (13)], we get a simple relation between the wavelet transform of $S_{d}$ and the Morse analyzing wavelet $\psi_{n} \alpha, \gamma$ :

$$
T_{\psi_{n, \gamma}}\left[S_{d}\right](b, a) \propto \frac{C_{n, \gamma}}{C_{n} \quad \alpha, \gamma}(2 \pi)^{\alpha} a^{\alpha}{ }^{1 / 2} \psi_{n} \quad \alpha, \gamma\left(\frac{b}{a}\right) .
$$

Then, the Morse wavelet-based power spectrum of $S_{d}$ [Eq. (19)] can be written as

$$
P_{S_{d}}^{\mathrm{CWT}}(f) \propto \frac{f_{0}^{2 \alpha}{ }^{1}}{f^{2 \alpha} C_{\psi}}\left[\frac{(2 \pi)^{\alpha} C_{n, \gamma}}{C_{n} \quad \alpha, \gamma}\right]^{2} \int_{\infty}^{+\infty}\left|\widehat{\psi}_{n} \alpha, \gamma(v)\right|^{2} d v .
$$

Given that we use the $\mathcal{L}^{2}$ norm, the integral in Eq. (22) is equal to one and we have

$$
P_{S_{d}}^{\mathrm{CWT}}(f) \propto \frac{f_{0}^{2 \alpha}{ }^{1}}{C_{\psi}}\left[\frac{(2 \pi)^{\alpha} C_{n, \gamma}}{C_{n} \alpha, \gamma}\right]^{2} f^{2 \alpha} .
$$

This analytical expression gives the following power-law behavior of the wavelet-based power spectrum of a fractional
Gaussian random signal $S_{d}$ with $\widehat{S}_{d}(f) \propto f \alpha$ :

$$
P_{S_{d}}^{\mathrm{CWT}}(f) \propto f^{\beta} \quad \text { with } \beta=2 \alpha .
$$

The fractional Gaussian random signal $S_{d}$ shown in Fig. 2(a) can be considered as a fractional integration of order $\alpha=0.25$ of a stationary zero mean Gaussian random signal with a flat spectrum (corresponding to a Hölder exponent $h=-0.5$, or equivalently to a monofractal signal of Hurst exponent of $H=h=-0.5$ ). The power spectrum of $S_{d}$ should, therefore, behave as $f^{2 \alpha}=f^{0.5}$. We show the power spectrum of $S_{d}$ computed from a standard FFT transformation $P_{S_{d}}^{\mathrm{FFT}}(f)$ in Fig. 2(b). The extraction of a power-law exponent from this spectrum is hampered by two limitations: (i) the frequencies of the spectrum are linearly distributed and not logarithmically, which necessitates an averaging of the FFT spectrum [as performed in Fig. 2(c)] in a logarithmic frequency scale, (ii) this spectrum is very noisy and the amplitude of this noise increases with the value of the frequency. The powerlaw exponent $\beta$ is estimated from a linear regression fit of the power spectra in logarithmic scales, as illustrated in Figs. 2(b) and 2(c) for $\log _{10} f \in[0,4]$ ).

When using the power spectrum computed with the wavelet transform $P_{S_{d}}^{\mathrm{CWT}}(f)$, the range of frequencies where the power-law can be estimated depends not only on the length of the signal, for a fixed sampling time, but also on 

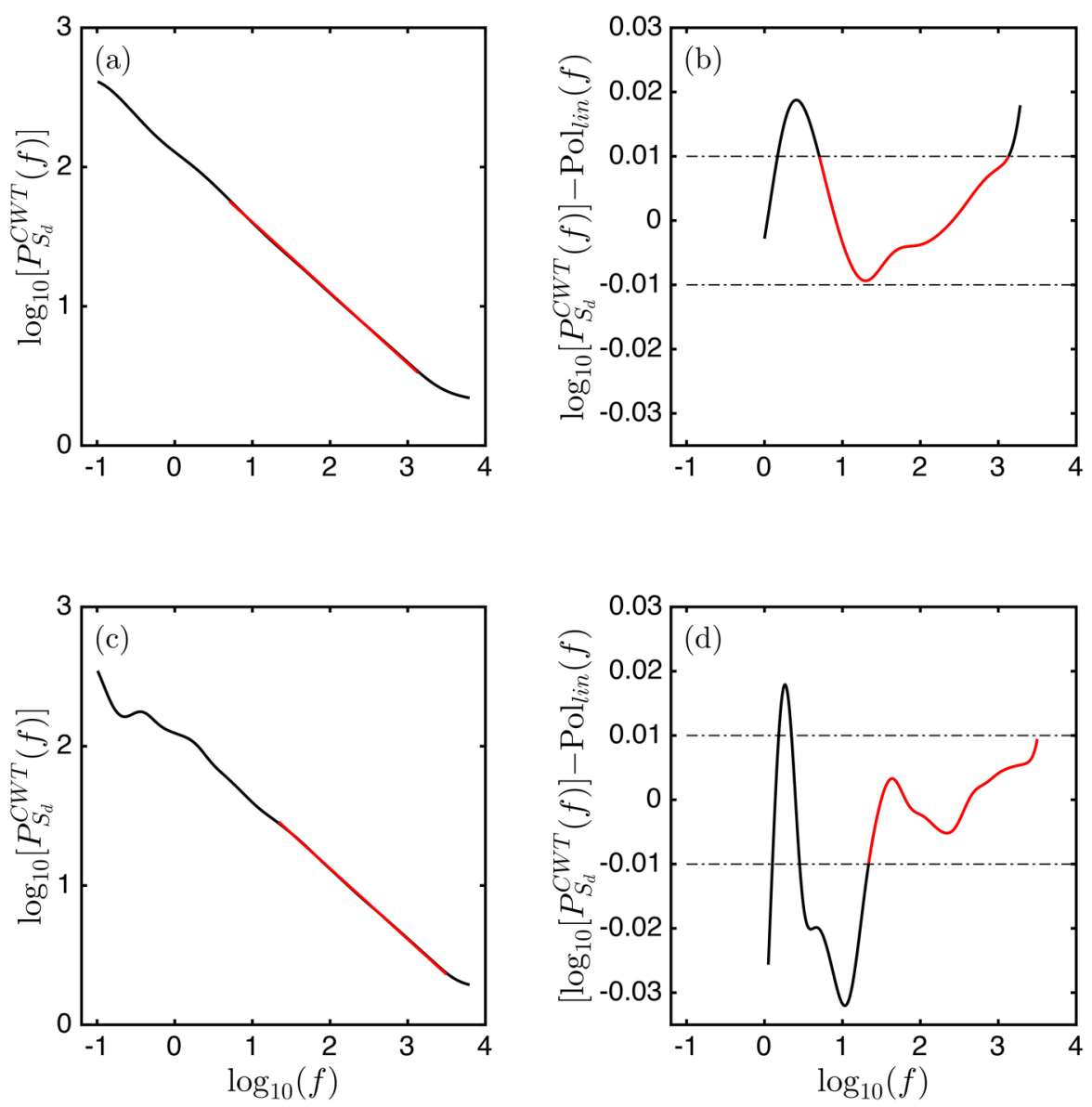

FIG. 6. Principle for the estimation of the $\beta$ exponent from wavelet based power spectra. Power spectra of the fractional Gaussian random signals shown in Fig. 5(a), computed with a Morse wavelet of exponent $\gamma \quad 1$, and $n \quad 1$ (a) and (b), and $n \quad 4$ (c) and (d). The range of frequency was maximized so that the difference of the power spectrum with its linear fit (b) and (d) remains in the interval [ $0.01,0.01]$. The result of this parametrization is highlighted with red curves in (a) and (d).

the analyzing wavelet. The Morse wavelets [Eq. (13)] are particularly interesting because by varying a single parameter $n$, one can modify their number of oscillations and hence one may privilege either periodic or singular behavior detection. This effect is illustrated in Figs. 3 and 4 for $n=1$ and $n=4$, respectively, $\gamma=1$ being fixed. Let us recall that $f_{0}=(n / \gamma)^{1 / \gamma} /(2 \pi)$, corresponding to the peak frequency of $\hat{\psi}_{n, \gamma}(2 \pi f)$, depends on both $n$ and $\gamma$. The scale $a$ of the wavelet is inversely proportional to its frequency: $a=f_{0} / f$. Given that the original signal $S_{d}$ is Gaussian, its wavelet transform coefficients at different scales are also Gaussian variables. As illustrated in Figs. 3(e) and 4(e) for the real part of the wavelet transform, the different p.d.fs computed at different scales (inverse of frequency) all superimpose on the same Gaussian distribution when rescaling the wavelet coefficients computed at a given scale $a$ by the standard deviation $\sigma(a)$ (the frequency dependence of the power-spectrum of $S_{d}$ can be obtained by computing the variance of the wavelet transform coefficients across scales $\sigma^{2}(a)\left(a=f_{0} / f\right)$ [Eqs. (18) and (19)]. This illustrates the advantage of the wavelet transform analysis with respect to Fourier analysis, since it provides a very efficient way to diagnose and characterize the monofractal (vs multifractal) nature of the analyzed signal with fluctuation statistics that do not (vs do) change across scales.
The modulus of the wavelet transforms is color coded in Figs. 3(f) and 4(f), after rescaling each line of the matrix $\left|T_{\psi}\left[S_{d}\right](b, a)\right|$ in between 0 and 1 to highlight their transformation when increasing the analyzing wavelet parameter $n$. For the smaller $n$ value $(n=1)$, the local maxima of the wavelet transform modulus follow almost vertical maxima lines, pointing toward the singular points of the signal, over a wide range of scales (frequencies) [Fig. 3(f)] allowing to better estimate and quantify the scaling properties of the analyzed signal. For larger $n$ value $(n=4)$, this range of scales (frequencies) shrinks to smaller (respectively higher) scales (respectively frequencies), leaving room to detect low frequency trends in the signal (if any). This suggests that to characterize properly singular power-law behavior from complex signals, it will be preferable to use Morse wavelets with $n$ as small as possible. On the contrary, to track periodic behavior, or more generally rhythms, higher $n$ values will be better suited. This conclusion is corroborated by the reconstruction of the power spectra from the modulus square of the wavelet transforms in Fig. 5.

The estimation of the local slope $\beta$ from the wavelet-based power spectra in logarithmic scales was performed by a homemade program under MATLAB environment. The principle of this computation is briefly outlined in Fig. 6. We looked for the largest frequency range $\left[\log _{10}\left(f_{\min }\right), \log _{10}\left(f_{\max }\right)\right]$ to 

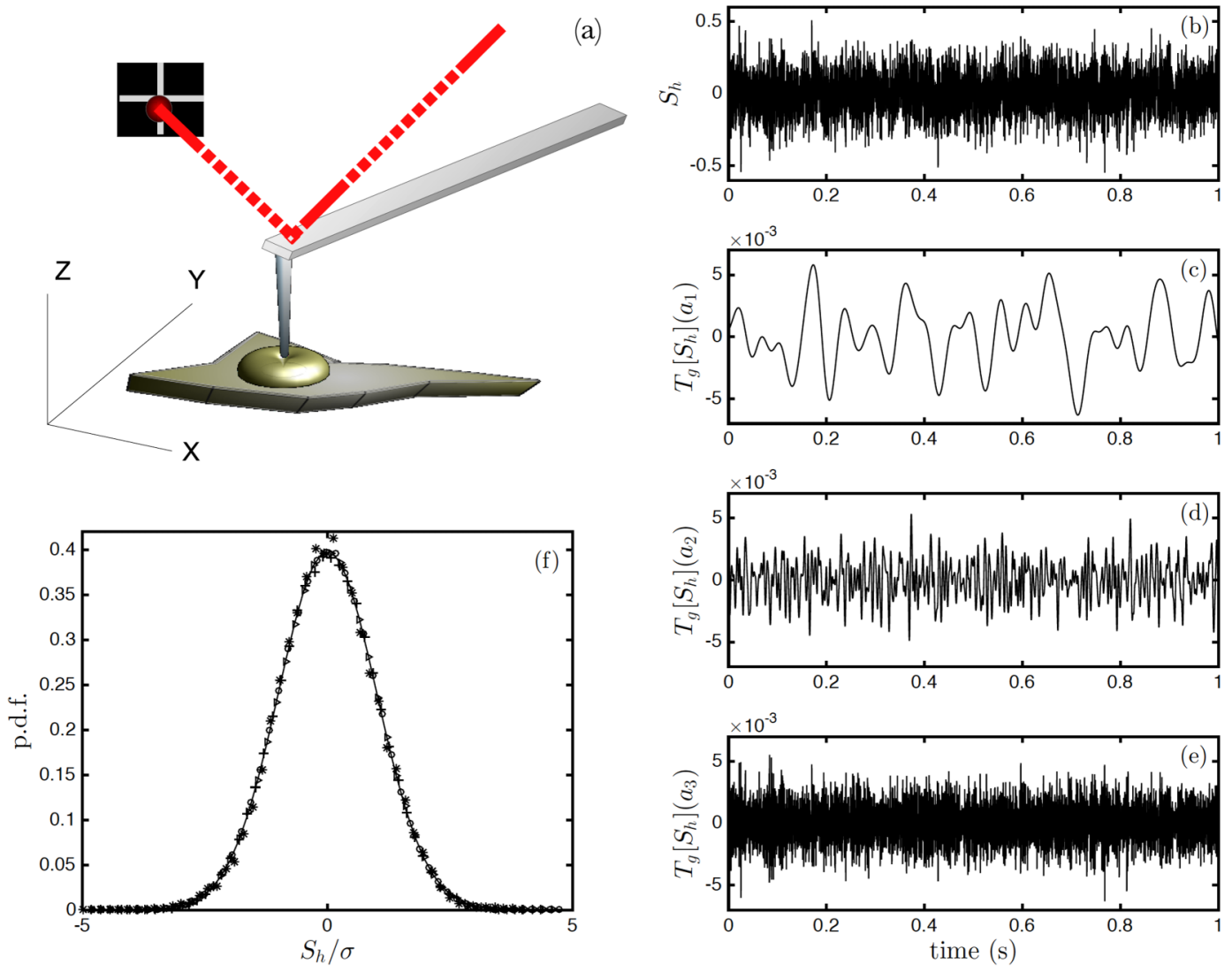

FIG. 7. (a) Sketch of the AFM principle. (b) Cantilever tip fluctuations $S_{d}$ recorded from a normal C2C12 myoblast with a loading force of $1 \mathrm{nN}$. [(c), (d), and (e)] Real part of the wavelet transform of $S_{d}: R_{\psi}(t, a) \quad \operatorname{Re}\left[T_{\psi}\left[S_{d}\right](t, a)\right]$ for three different scale values $a_{i} \quad f_{0} / f_{i}$ with $f_{1} \quad 10 \mathrm{~Hz}(\circ), f_{2} \quad 10^{2} \mathrm{~Hz}(*)$, and $f_{3} 10^{3} \mathrm{~Hz}(\triangleright)$. (f) P.d.fs of $R_{\psi}(., a) / \sigma(a)$ for the three scale values shown in (c), (d) and (e) after rescaling by the corresponding standard deviation $\sigma(a)$. The solid line corresponds to the rescaled p.d.f. of $S_{d}$. The analyzing wavelet is a Morse wavelet of exponents $\gamma \quad 1, n \quad 1$ [Eq. (13)].

perform a linear regression fit of the power spectrum (in log scales) to get some estimate of the power-law exponent $\beta$. This was done by subtracting the so-obtained linear fit from the power spectrum and by requiring that the local distance of the power spectrum with its linear parametrization remained in the $[-0.01,0.01]$ interval [Figs. 6(b) and 6(d)]. The choice of the order $n$ of the Morse wavelet is important with respect to that issue. With $n=1$ [Figs. 6(a) and 6(b)], we get a slope $\beta_{2}=0.507$ over a frequency interval $\log _{10}[f] \in[0.04,2.09]$, which is significantly wider than the frequency interval $\log _{10}[f] \in[0.40,1.93]$ delimited with $n=4$ [Figs. 6(c) and 6(d)]. The relevance of the $\beta$ slope estimated from the power spectrum will be reinforced or weakened according to whether the scaling law occurs over a wider or narrower frequency range. This methodology proved to be very useful and efficient to define the frequency range (experimental situations) where cell rheology obeys a fractional behavior.

\section{RESULTS AND DISCUSSION}

\section{A. Creep fluctuation signals recorded from living muscle precursor cells have Gaussian statistics across scales}

The principle of the AFM experiment and a cantilever position fluctuation signal $S_{\mathrm{d}}$ recorded from an adherent living C2C12 myoblast are shown in Figs. 7(a) and 7(b), respectively. In Figs. 7(c) and 7(e), the real part of the wavelet transform $R_{\psi}(t, a)=\operatorname{Re}\left[T_{\psi}\left[S_{d}\right]\left(t, a_{i}\right)\right]$ is plotted for different wavelet scales $a_{i}=f_{0} / f_{i}$ over a $1 \mathrm{~s}$ time interval. As shown in Fig. 7(f), the corresponding p.d.f.s of these wavelet coefficients have a Gaussian shape at the three considered scales. Importantly, these p.d.f.s superimpose on a single curve when rescaling $R_{\psi}\left(., a_{i}\right)$ by its standard deviation $\sigma\left(a_{i}\right)$. This demonstrates that these AFM tip fluctuations are self-similar and that the signal $S_{d}$ is monofractal [50,51]. As reported in Fig. 8, we have reproduced this scaling analysis on different muscle precursor cells (C2C12 myoblasts), cultured in different conditions, namely, normal culture medium [Figs. 8(a) 8(c)], ATP deprived cells [Figs. 8(d) 8(f)], and blebbistatin treated cells [Figs. 8(g) 8(i)]. We confirm in Figs. 8(a), 8(d), and 8(g) that these fluctuations remain Gaussian in the wavelet frequency range: $f=f_{0} / a \in[5 \mathrm{~Hz} 150 \mathrm{~Hz}]$, and independently of the cell treatment, in agreement with previous experimental evidences obtained with single particle tracing [101,102]. More importantly, we demonstrate the monofractal scaling properties of these fluctuations by superimposing their p.d.fs computed at different wavelet scales, after rescaling $\mathcal{R}_{\psi}\left(t, f_{0} / f\right)$ by its standard deviation $\sigma(f)$. This wavelet-based time frequency decomposition of $S_{d}$ was thus mandatory to validate that these rheological fluctuation signals are self-similar with Gaussian statistics, and that they can be described by a single exponent $\alpha$, which can be estimated from the power-law decay of their power spectra $P_{S_{\mathrm{d}}}^{\mathrm{CWT}}(f)$ [Eq. (24)]. 

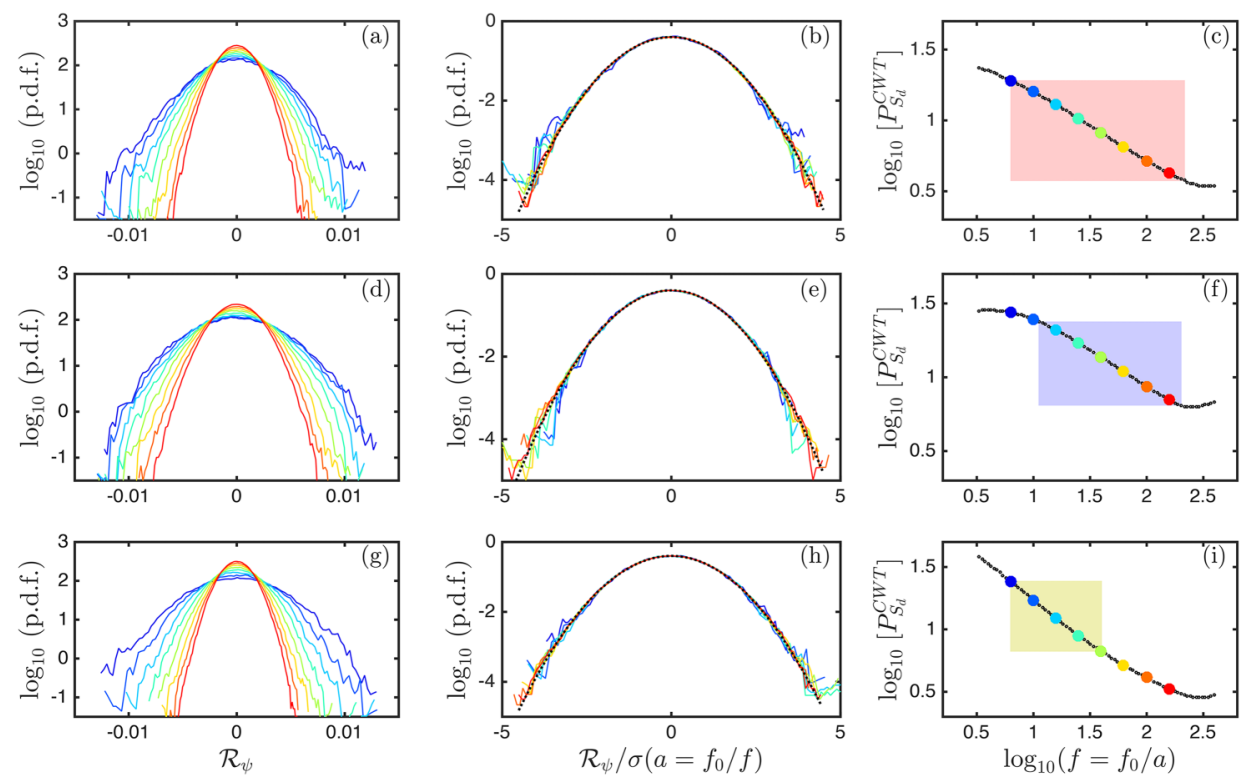

FIG. 8. Characterizing the AFM cantilever position fluctuations $S_{\mathrm{d}}$ and their scale invariance properties. [(a), (b), and (c)] Normal C2C12 myoblast cell. [(d), (e), and (f)] ATP depleted C2C12 myoblast cell. [(g), (h), and (i)] Blebbistatin treated C2C12 myoblast cell. [(a), (d), and (g)] P.d.fs of the real part of the wavelet transform $R_{\psi}(., a)$ of the signal $S_{\mathrm{d}}$ (logarithmic representation) computed with a Morse analyzing wavelet of exponents $\gamma \quad 1, n \quad 1$ [Eq. (13)] for different wavelet scales $a_{i} \quad f_{0} / f$ with $f \quad 6.8 \mathrm{~Hz}$ (dark blue), $f \quad 10 \mathrm{~Hz}$ (blue), $f \quad 15.8 \mathrm{~Hz}$ (light blue), $f \quad 25.1 \mathrm{~Hz}$ (cyan), $f \quad 39.8 \mathrm{~Hz}$ (green), $f \quad 63.1 \mathrm{~Hz}$ (yellow), $f \quad 100 \mathrm{~Hz}$ (light red), $f \quad 158.5 \mathrm{~Hz}$ (red). [(b), (e), and (h)] P.d.fs of $R_{\psi}(., a) / \sigma(a)$, where $\sigma(a)$ is the standard deviation. [(c), (f), and (i)] $\log _{10}\left[P_{S_{\mathrm{d}}}^{\mathrm{CWT}}(f)\right]$ vs $\log _{10}(f)$ computed from the wavelet transform of the rheological fluctuation signals $S_{\mathrm{d}}$ [Eqs. (23) and (24)]; the color dots correspond to the scales $a_{i}$ defined in (a) (d), and (g); the color shaded rectangles delimit the frequency ranges where the power spectrum has been identified to behave as $P_{S_{d}}^{C W T}(f) \sim f^{-\beta}$.

\section{B. Comparing the fractional rheology of five different sets of muscle precursor cells}

We developed a home-made software, described in Sec. II B 2 [Figs. 5 and 6], to delimit the range of $f$ [color shaded rectangles in Figs. 8(c), 8(f), and 8(i)] where a power-law exponent can be recognized from $P_{S_{d}}^{C W T}(f)$. We notice in Fig. 8(i), corresponding to a myoblast cell treated with blebbistatin, that the range of $f$ has shrunk to less than a decade and shifted to lower frequencies, as compared to a normal myoblast. Also, the corresponding yellow rectangle has moved to larger $\log _{10}\left[P_{S_{d}}^{\mathrm{CWT}}\right]$ values, as the signature of greater fluctuation amplitude. This is an indication that the amount of mechanical energy transferred from the surrounding liquid medium to the CSK is larger for this blebbistatin treated myoblast cell and that this cell is softer than the normal myoblast shown in Fig. 8(c). The slope $\beta=2 \alpha$ computed by linear regression fit inside the shaded rectangles of Figs. 8(c), 8(f), and 8(i) corresponds to the following estimates of the rheological exponent $\alpha=0.24 \pm 0.01,0.23 \pm 0.01$ and $0.34 \pm 0.01$, respectively. The fractional exponent $\alpha$ for the blebbistatin treated myoblast is significantly larger, which confirms that this cell is not only softer but also behaves as more viscous than the normal and the ATP depleted myoblasts studied in this same Fig. 8.

Figure 9 generalizes the shaded rectangle representation of Figs. 8(c), 8(f), and 8(i) to compare the rheology of five different sets of $\mathrm{C} 2 \mathrm{C} 12$ and human myoblasts and one set of differentiated $\mathrm{C} 2 \mathrm{C} 12$ myotubes from myoblasts. Each rectangle corresponds to an independent experiment. Figure 9(a) concentrates on $\mathrm{C} 2 \mathrm{C} 12$ myoblasts (normal in red, ATP deprived in blue and fixed in green). The fractional exponent $\alpha=\beta / 2$ [Eq. (24)] is introduced as a third coordinate to better separate the different cell types. The mean values obtained when averaging over 2530 cells of each type confirm that the normal $(\bar{\alpha}=0.24 \pm 0.01)$ and ATP deprived myoblasts $(\bar{\alpha}=0.22 \pm 0.01)$ behave rather similarly, with fractional fluctuations extending over more than a decade $\left(\Delta\left[\log _{10} f\right] \geq 1.15\right)$. ATP deprived cells do not loose their global flat and elongated shape [Fig. 9(b)], but the perinuclear actin stress fibers are replaced by actin aggregates which may also act as actin CSK cross-linkers. Fixing the myoblasts is a dramatic and irreversible procedure that kills the cells, however its preserves (only partly) their CSK and reinforces the remaining actin filament cross-linked structure with PFA $(\bar{\alpha}=0.26 \pm 0.01)$. This treatment was also used before staining the cells for the fluorescence microscopy images shown in Fig. 1. Figure 9(b) compares the normal buffered $\mathrm{C} 2 \mathrm{C} 12$ myoblasts (red), with blebbistatin treated $\mathrm{C} 2 \mathrm{C} 12$ myoblasts (yellow), $\mathrm{C} 2 \mathrm{C} 12$ myotubes (violet) and human DMD myoblasts (gray). It is quite difficult to distinguish the normal $\mathrm{C} 2 \mathrm{C} 12$ myoblasts $(\bar{\alpha}=0.24 \pm 0.01)$ from the human DMD myoblasts $(\bar{\alpha}=0.24 \pm 0.01)$. However, we must mention that for a non-negligible fraction $(22 \%)$ of DMD human myoblasts, the extraction of a power-law regime was very difficult, probably as the consequence of an immediate cell shape change. Thus, in this latter case, the statistical sampling was limited. The $\mathrm{C} 2 \mathrm{C} 12$ myotubes, which were differentiated from the myoblasts, behave with a larger exponent $\alpha(\bar{\alpha}=0.29 \pm 0.02)$, over a narrower $f$ range [Figs. 9(c) and 9(d)]. Would the myotubes display different rheological properties? This conclusion could be reasonable, regarding the strong remodelling of their cytoskeleton, as compared to their precursors (Fig. 1). One 
(a)

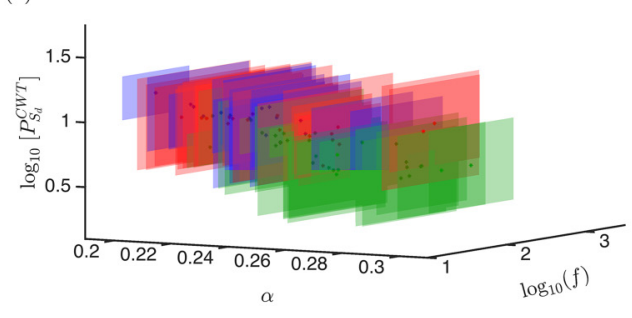

(b)

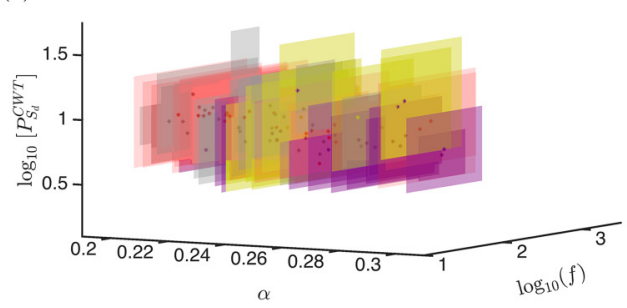

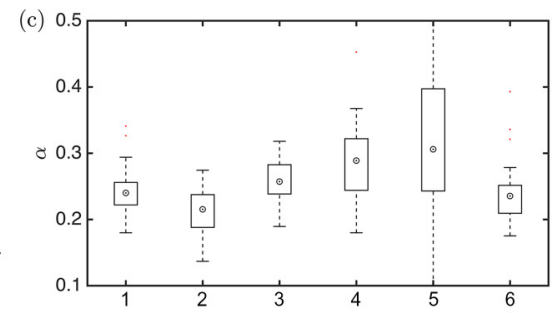

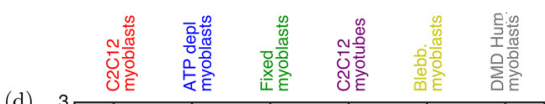

(d)

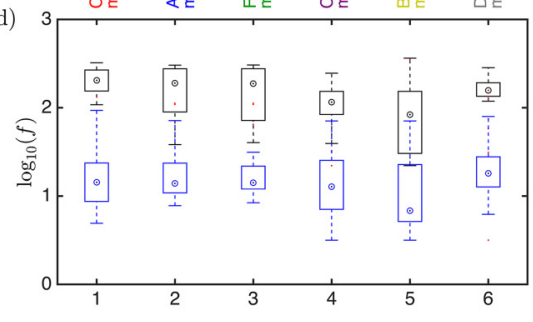

FIG. 9. $3 \mathrm{D}$ color shaded rectangles illustrating the ranges of frequency $\left[\log _{10}(f)\right]$ and fluctuation energy (log $\left.\log _{10}\left[P_{S_{\mathrm{d}}}^{\mathrm{CWT}}\right]\right)$ values where fractional behavior with exponent $\alpha$ has been identified. (a) Comparison of normal (red) with ATP depleted (blue), and fixed (green) C2C12 myoblasts. (b) Comparison of normal C2C12 myoblasts (light red), blebbistatin treated C2C12 myoblasts (yellow), human DMD myoblasts (grey), and C2C12 myotubes (violet). (c,d) Boxplot repre sentation of $\alpha \quad \beta / 2$ (c), and of the inferior (blue), and superior (black) frequency [ $\left.\log _{10}(f)\right]$ limits (d), where $\alpha$ has been estimated, for each cell type. 2530 cells were tested for each case.

could be tempted to compare the myotubes with the fixed myoblast cells, since their scaling exponent and frequency range are similar. However, let us point out that the fluctuation energy (the amplitude of the power spectrum) is significantly larger for the myotubes, indicating that they are softer than myoblasts. Indeed, their actin CSK is very different [Figs. 1(a) and 1(c)], and their mechanical response is different too. Finally, the blebbistatin treated myoblasts also behave with a larger $\alpha$ exponent $(\bar{\alpha}=0.31 \pm 0.02)$, on a quite variable frequency range, and their fluctuation energy is even higher than previously observed for the myotubes. These cells are less elastic (the greater $\alpha$, the greater the viscous component), and less tensed that normal myoblasts, their CSK has lost in part or totally its cross-linked architecture typical of prestressed cells on adherent supports (Fig. 1).

\section{CONCLUSION}

To conclude, we have proposed an original method, based on complex wavelet transform decomposition of rheological signals, to reveal the occurrence of fractional fluctuations and estimate accurately the power-law exponent $\alpha$ as a signature of self-similar scale-invariant mechanics of living cells. This method was applied to creep experiments performed with an AFM nanoindenter placed in contact with single myoblasts and myotubes adherent on collagen coated coverslips. This methodology was tested on limited statistical samples $(\sim 25$ 30 cells for each case), but it has demonstrated its efficiency and its expeditiousness on rather short time recording $(\leqslant 100 \mathrm{~s})$. We have not considered lower frequencies $(<5$ $\mathrm{Hz}$ ) in that work, not only because they would have required longer time series, but also to avoid active actin CSK remodeling processes driven by molecular motors (myosin-II) to be involved. We hope to elaborate on the study of active driven rheological properties of living cells in a future work.

\section{ACKNOWLEDGMENTS}

The authors thank L. Berguiga, B. Laperrousaz, C. Martinez-Torres, O. Naimark, F. Perez-Reche, and S. Polizzi for fruitful discussions. The authors are very grateful to T. Muller and to the JPK R\&D department for their partnership. L.S., A.A., and F.A. acknowledge support from the Laboratory of Physics (ENS Lyon/CNRS) and from the Agence Nationale de la Recherche ANR-10-BLAN-1516 "Mechastem". The Ph.D. scholarship of L.Streppa was supported by AFM Téléthon. E.G.C.'s work was funded by the President of Russia Grant No. 14.W01.17.2674-MK for young scientists and by the Metchnikov Program.

\section{References}

[1] Gardel, M. L., K. E. Kasza, C. P. Brangwynne, J. Liu, and D. A. Weitz, "Mechanical response of cytoskeletal networks," Methods Cell Biol. 89, 487519 (2008).

[2] Lieleg, O., M. M. A. E. Claessens, and A. R. Bausch, "Structure and dynamics of cross linked actin networks," Soft Matter 6, 218225 (2010).

[3] Blanchoin, L., R. Boujemaa Paterski, C. Sykes, and J. Plastino, "Actin dynamics, architecture and mechanics in cell motility," Physiol. Rev. 94, 235263 (2014).

[4] Murrell, M., P. W. Oakes, M. Lenz, and M. L. Gardel, "Forcing cells into shape: the mechanics of actomyosin contractility," Nat. Rev. Mol. Cell. Biol. 16, 486498 (2015).

[5] Hoffman, B. D., and J. C. Crocker, "Cell mechanics: Dissecting the physical responses of cells to force," Annu. Rev. Biomed. Eng. 11, 259288 (2009).

[6] Brunner, C., A. Niendorf, and J. A. Kas, "Passive and active single cell biomechanics: A new perspective in cancer diagnosis," Soft Matter 5, 21712178 (2009).

[7] Mofrad, M. R., "Rheology of the cytoskeleton," Annu. Rev. Fluid Mech. 41, 433453 (2009). 
[8] Chauviere, A., L. Preziosi, and C. Verdier, Cell Mechanics: From Single Scale Based Models to Multiscale Modeling (Chapman \& Hall/CRC, Boca Raton, 2010).

[9] Kollmannsberger, P., and B. Fabry, "Linear and nonlinear rheology of living cells," Annu. Rev. Mater. Res. 41, 7597 (2011).

[10] Rodriguez, M. L., P. J. McGarry, and N. J. Sniadecki, "Review on cell mechanics: Experimental and modeling approaches," Appl. Mech. Rev. 65, 60801 (2013).

[11] Laperrousaz, B., G. Drillon, L. Berguiga, F. Nicolini, B. Audit, V. Maguer Satta, A. Arneodo, and F. Argoul, "From elasticity to inelasticity in cancer cell mechanics: A loss of scale invariance," AIP Conf. Proc. 1760, 020040 (2016).

[12] Laperrousaz, B., L. Berguiga, F. E. Nicolini, C. Martinez Torres, A. Arneodo, V. Maguer Satta, and F. Argoul, "Revealing stiffening and brittlening of chronic myelogenous leukemia hematopoietic primary cells through their temporal response to shear stress," Phys. Biol. 13, 03LT01 (2016).

[13] Streppa, L., A. Devin, L. Schaeffer, A. Arneodo, and F. Argoul, "Prestressed cells are prone to cytoskeleton failures under localized shear strain: An experimental demonstration on muscle precursor cells," Sci. Rep. 8, 8602 (2018).

[14] Broedersz, C. P., and F. C. Mackintosh, "Modeling semiflexible polymer networks," Rev. Mod. Phys. 86, 9951036 (2014).

[15] Rigato, A., A. Miyagi, S. Scheuring, and F. Rico, "High frequency microrheology reveals cytoskeleton dynamics in living cells," Nat. Phys. 13, 771775 (2017).

[16] Broedersz, C. P., M. Depken, N. Y. Yao, M. R. Pollak, D. A. Weitz, and F. C. MacKintosh, "Cross link governed dynamics of biopolymer networks," Phys. Rev. Lett. 105, 238101 (2010).

[17] Espinosa, G., I. Lopez Montero, F. Monroy, and D. Langevin, "Shear rheology of lipid monolayers and insights on membrane fluidity," Proc. Natl. Acad. Sci. USA 108, 60086013 (2011).

[18] Deseri, L., P. Pollaci, M. Zingales, and K. Dayal, "Fractional heredi tariness of lipid membranes: Instabilities and linearized evolution," J. Mechan. Behav. Biomed. Mater. 58, 1127 (2016)

[19] Salbreux, G., G. Charras, and E. Paluch, "Actin cortex mechanics and cellular morphogenesis," Trends Cell Biol. 22, 53645 (2012)

[20] Gittes, F., B. Schnurr, P. D. Olmsted, F. C. MacKintosh, and C. F. Schmidt, "Microscopic viscoelasticity: Shear moduli of soft materials determined from thermal fluctuations," Phys. Rev. Lett. 79, 32863289 (1997).

[21] Mason, T. G., "Estimating the viscoelastic moduli of complex fluids using the generalized Stokes Einstein equation," Rheol. Acta. 39, 371378 (2000).

[22] Evans, E., M. E. Cates, and P. Sollich, "Diffusion and rheology in a model of glassy materials," Eur. Phys. J. B 10, 705718 (1999).

[23] Pottier, N., and A. Mauger, "Anomalous diffusion of a particle in an aging medium," Physica A 332, 1528 (2004).

[24] Abou, B., F. Gallet, P. Monceau, and N. Pottier, "Generalized Einstein relation in an aging colloidal glass," Physica A 387, 34103422 (2008).

[25] Basu, A., J. F. Joanny, F. Jülicher, and J. Prost, "Thermal and non thermal fluctuations in active polar gels," Eur. Phys. J. E 27, 149160 (2008).

[26] Muzy, J. F., and E. Bacry, "Multifractal stationary random measures and multifractal random walks with $\log$ infinitely divisible scaling laws," Phys. Rev. E 66, 056121 (2002).

[27] Pan, S., Z. W. Wu, W. H. Wang, M. Z. Li, and L. Xu, "Structural origin of fractional Stokes Einstein relation in glass forming liquids," Sci. Rep. 7, 39938 (2017).
[28] Radmacher, M., "Measuring the elastic properties of living cells by the atomic force microscope," Methods Cell Biol. 68, 6790 (2002).

[29] Mahaffy, R. E., S. Park, E. Gerde, J. Käs, and C. K. Shih, "Quantitative analysis of the viscoelastic properties of thin regions of fibroblasts using atomic force microscopy," Biophys. J. 86, 17771793 (2004).

[30] Abidine, Y., V. Laurent, R. Michel, A. Duperray, I. L. Palade, and C. Verdier, "Physical properties of polyacrylamide gels probed by AFM and rheology," Europhys. Lett. 109, 38003 (2015).

[31] Martinez Torres, C., A. Arneodo, L. Streppa, P. Argoul, and F. Argoul, "Passive microrheology of soft materials with atomic force microscopy: A wavelet based spectral analysis," Appl. Phys. Lett. 108, 034102 (2016).

[32] Lyapunova, E., A. Nikituk, Y. Bayandin, O. Naimark, C. Rianna, and M. Radmacher, "Passive microrheology of normal and cancer cells after ML7 treatment by atomic force microscopy," AIP Conf. Proc. 1760, 020046 (2016).

[33] Nambiar, R., R. E. McConnell, and M. J. Tyska, "Control of cell membrane tension by myosin I," Proc. Natl. Acad. Sci. USA 106, 1197211977 (2009).

[34] Grossmann, A., and J. Morlet, "Decomposition of Hardy functions into square integrable wavelets of constant shape," SIAM J. Math. Anal. 15, 723736 (1984).

[35] Wavelets and their Applications, edited by Y. Meyer (Springer, Berlin, 1992).

[36] Daubechies, I., Ten Lecture on Wavelets (SIAM, Philadelphia, 1992).

[37] Arneodo, A., F. Argoul, E. Bacry, J. Elezgaray, and J. F. Muzy, Ondelettes, Multifractales et Turbulences: de l'ADN aux croissances cristallines (Diderot Editeur, Art et Sciences, Paris, 1995).

[38] Holschneider, M., Wavelets: An Analysis Tool (Oxford University, Oxford, 1996).

[39] Abry, P., Ondelettes et Turbulences (Diderot Editeur, Arts et Sciences, Paris, 1997).

[40] Mallat, S., A Wavelet Tour of Signal Processing (Academic, New York, 1998).

[41] Torresani, B., Analyse Continue par Ondelettes (Editions de Physique, Les Ulis, 1998).

[42] Flandrin, P., Time Frequency/Time Scale Analysis (Academic, New York, 1999).

[43] Arneodo, A., B. Audit, N. Decoster, J. F. Muzy, and C. Vaillant, "Wavelet based multifractal formalism: application to DNA sequences, satellite images of the cloud structure an stock market data," in The Science of Disasters: Climate Disruptions, Heart Attacks and Market Crashes, edited by A. Bunde, J. Kropp, and H. J. Schellnhuber (Springer Verlag, Berlin, 2002), pp. 26102.

[44] Arneodo, A., B. Audit, C. Moskalenko, J. Moukhtar, C. Vaillant, F. Argoul, Y. D'Aubenton Carafa, and C. Thermes, "From DNA sequence to chromatin organization: The fundamental role of genomic long range correlations," Bulletin de l'Académie Royale de Belgique, Mémoire de la Classe des Sciences Tome XXVII, 2049 (2008).

[45] Arneodo, A., C. Vaillant, B. Audit, F. Argoul, Y. d'Aubenton Carafa, and C. Thermes, "Multi scale coding of genomic information: From DNA sequence to genome structure and function," Phys. Rep. 498, 45188 (2011).

[46] Gabor, D., "Theory of communication," J. Inst. Electr. Eng. 93, 429457 (1946).

[47] Muzy, J. F., E. Bacry, and A. Arneodo, "Wavelets and multifractal formalism for singular signals: Application to turbulence data," Phys. Rev. Lett. 67, 35153518 (1991). 
[48] Bacry, E., J. F. Muzy, and A. Arneodo, "Singularity spectrum of fractal signals from wavelet analysis: Exact results," J. Stat. Phys. 70, 635674 (1993).

[49] Muzy, J. F., E. Bacry, and A. Arneodo, "Multifractal formalism for fractal signals: The structure function approach versus the wavelet transform modulus maxima method," Phys. Rev. E 47, 875884 (1993).

[50] Muzy, J. F., E. Bacry, and A. Arneodo, "The multifractal formalism revisited with wavelets,” Int. J. Bifurcat. Chaos 4, 245302 (1994).

[51] Arneodo, A., E. Bacry, and J. Muzy, "The thermodynamics of fractals revisited with wavelets," Physica A 213, 232275 (1995).

[52] Arneodo, A., N. Decoster, P. Kestener, and S. G. Roux, "A wavelet based method for multifractal image analysis: From theoretical con cepts to experimental applications," Adv. Imaging Electron Phys. 126, 192 (2003).

[53] Gerasimova, E., B. Audit, S. G. Roux, A. Khalil, O. Gileva, F. Argoul, O. Naimark, and A. Arneodo, "Wavelet based multifractal analysis of dynamic infrared thermograms to assist in early breast cancer diagnosis," Front. Physiol. 5, 176 (2014).

[54] Gerasimova Chechkina, E., B. Toner, Z. Marin, B. Audit, S. G. Roux, F. Argoul, A. Khalil, O. Gileva, O. Naimark, and A. Arneodo, "Comparative multifractal analysis of dynamic infrared thermograms and X Ray mammograms enlightens changes in the environment of malignant tumors," Front. Physiol. 7, 336 (2016).

[55] Digiuni, S., A. Berne Dedieu, C. Martinez Torres, J. Szecsi, M. Bendahmane, A. Arneodo, and F. Argoul, "Single cell wall non linear mechanics revealed by a multi scale analysis of AFM force indentation curves,” Biophys. J. 108, 22352248 (2015).

[56] Arneodo, A., B. Audit, P. Kestener, and S. Roux, "Wavelet based multifractal analysis," Scholarpedia 3, 4103 (2008).

[57] Roland, T., A. Khalil, A. Tanenbaum, L. Berguiga, P. Delichère, L. Bonneviot, J. Elezgaray, A. Arneodo, and F. Argoul, "Revisiting the physical processes of vapodeposited thin gold films on chemically modified glass by atomic force and surface plasmon microscopies," Surf. Sci. 603, 33073320 (2009).

[58] Khalil, A., J. L. Grant, L. B. Caddle, E. Atzema, K. D. Mills, and A. Arneodo, "Chromosome territories have a highly nonspherical morphology and nonrandom positioning," Chromosome Res. 15, 899916 (2007).

[59] Martinez Torres, C., L. Berguiga, L. Streppa, E. Boyer Provera, L. Schaeffer, J. Elezgaray, A. Arneodo, and F. Argoul, "Diffraction phase microscopy: Retrieving phase contours on living cells with a wavelet based space scale analysis," J. Biomed. Opt. 19, 36007 (2014).

[60] Martinez Torres, C., B. Laperrousaz, L. Berguiga, E. Boyer Provera, J. Elezgaray, F. E. Nicolini, V. Maguer Satta, A. Arneodo, and F. Argoul, "Deciphering the internal complexity of living cells with quantitative phase microscopy: a multiscale approach," J. Biomed. Opt. 20, 096005 (2015).

[61] Deseri, L., M. Di Paola, and M. Zingales, "Free energy and states of fractional order hereditariness," Int. J. Solids Struct. 51, 31563167 (2014).

[62] Szustakowski, J. D., J. H. Lee, C. A. Marrese, P. A. Kosinski, N. Nirmala, and D. M. Kemp, "Identification of novel pathway regu lation during myogenic differentiation," Genomics 87, 129138 (2006).

[63] Mandel, L., D. J. Benos, F. Bronner, and A. Leinzeller, "Energy metabolism of cellular activation, growth and transforma tion," in Current Topics in Membranes and Transport: Role of Membranes in Cell Growth and Differentiation (Academic, 1986), pp. 261279.
[64] Nakayama, K., F. Okamoto, and Y. Harada, "Antimycin $\{$ A $\}$ : Isolation from a new $\{\mathrm{S}\}$ treptomyces and activity against rice plant blast fungi," J. Antibiot. 9, 6366 (1956).

[65] Bright, R. T., C. G. Salvaterra, L. J. Rubin, and X. J. Yuan, "Inhibition of glycolysis by $2 \mathrm{DG}$ increases $\left[\mathrm{Ca}^{2+}\right](\mathrm{i})$ in pulmonary arterial smooth muscle cells," Am. J. Physiol. 269, L203 (1995).

[66] Theander, S., D. P. Lew, and O. Nüuße, "Granule specific ATP requirements for $\mathrm{Ca}^{2+}$ induced exocytosis in human neutrophils. Evidence for substantial ATP independent release," J. Cell Sci. 115, 29752983 (2002).

[67] Allingham, J. S., R. Smith, and I. Rayment, "The structural basis of blebbistatin inhibition and specificity for myosin II,” Nat. Struct. Mol. Biol. 12, 378379 (2005).

[68] Griffin, M. A., S. Sen, H. L. Sweeney, and D. E. Discher, "Adhesion contractile balance in myocyte differentiation," J. Cell Sci. 117, 58555863 (2004).

[69] Sader, J. E., I. Larson, P. Mulvaney, and L. R. White, "Method for the calibration of atomic force microscope cantilevers," Rev. Sci. Instrum. 66, 37893798 (1995).

[70] Sader, J. E., J. A. Sanelli, B. D. Adamson, J. P. Monty, X. Wei, S. A. Crawford, J. R. Friend, I. Marusic, P. Mulvaney, and E. J. Bieske, "Spring constant calibration of atomic force microscope cantilevers of arbitrary shape," Rev. Sci. Instrum. 83, 103705 (2012).

[71] Stark, R. W., T. Drobek, and W. M. Heckl, "Thermomechanical noise of a free $\mathrm{v}$ shaped cantilever for atomic force microscopy," Ultramicroscopy 86, 207215 (2001).

[72] Djordjevic, V. D., J. Jaric, B. Fabry, J. J. Fredberg, and S. Dimitrije, "Fractional derivatives embody essential features of cell rheological behavior," Ann. Biomed. Eng. 31, 692699 (2003).

[73] Sneddon, I. N., "The relation between load and penetration in the axi symmetric Boussinesq problem for a punch of arbitrary profile," Int. J. Eng. Sci. 3, 4757 (1965).

[74] Cheng, Y. T., and C. M. Cheng, "Scaling, dimensional analysis, and indentation measurements," Mater. Sci. Eng.: R: Rep. 44, 91149 (2004).

[75] Cheng, Y. T., and F. Yang, "Obtaining shear relaxation modulus and creep compliance of linear viscoelastic materials from instrumented indentation using axisymmetric indenters of power law profiles," J. Mater. Res. 24, 30133017 (2009).

[76] Alotta, G., O. Barrera, A. C. Cocks, and M. D. Paola, "On the behav ior of a three dimensional fractional viscoelastic constitutive model," Meccanica 52, 21272142 (2017).

[77] Nutting, P., "A new general law of deformation," J. Franklin Inst. 191, 679685 (1921).

[78] Di Paola, M., A. Pirrotta, and A. Valenza, "Visco elastic behavior through fractional calculus: An easier method for best fitting experi mental results," Mech. Mater. 43, 799806 (2011).

[79] Paola, M. D., and M. Zingales, "Exact mechanical models of frac tional hereditary materials," J. Rheol. 56, 9831004 (2012).

[80] Di Paola, M., F. P. Pinnola, and M. Zingales, "A discrete mechanical model of fractional hereditary materials," Meccanica 48, 15731586 (2013).

[81] Miller, K. S., and B. Ross, An Introduction to the Fractional Calculus and Fractional Differential Equations (Wiley Interscience, New York, USA, 1993).

[82] Combes, J., A. Grossmann, and P. Tchamitchian, Wavelets: Time Frequency Methods and Phase Space (Springer Verlag, Berlin, Heidelberg, 1989).

[83] Arneodo, A., G. Grasseau, and M. Holschneider, "Wavelet transform of multifractal,” Phys. Rev. Lett. 61, 22812284 (1988). 
[84] Holschneider, M., "On the wavelet transform of fractal objects," J. Stat. Phys. 50, 963993 (1988).

[85] Jaffard, S., "Holder exponents at given points and wavelet coefficients," Comptes Rendus de l'Académie des Sciences Série I 1308, 7981 (1989).

[86] Holschneider, M., and P. Tchamitchian, "Régularité locale de la fonction non différentiable de Riemann," in Les Ondelettes en 1989, edited by P. G. Lemarié (Springer, Berlin, 1990), pp. 102124.

[87] Jaffard, S., "Pointwise smoothness two microlocalization and wavelet coefficients," Publicacions Matematiques 35, 155168 (1991).

[88] Mallat, S., and W. L. Hwang, "Singularity detection and processing with wavelets," IEEE Trans. Inf. Theory 38, 617643 (1992).

[89] Carmona, R., W. L. Hwang, and B. Torresani, Practical Time Frequency Analysis (Academic, 1998).

[90] Jaffard, S., "Multifractal formalism for functions part I: Results valid for all functions," SIAM J. Math. Anal. 28, 944970 (1997).

[91] Jaffard, S., "Multifractal formalism for functions part II: Self similar functions," SIAM J. Math. Anal. 28, 971998 (1997).

[92] Morlet, J., G. Arenss, E. Fourgeau, and D. Giard, "Wave propagation and sampling theory Part I: Complex signal and scattering in multi layered media," Geophysics 47, 203221 (1982).

[93] Argoul, P., and T. P. Le, "Instantaneous indicators of structural behaviour based on the continuous cauchy wavelet analysis," Mech. Syst. Signal Process. 17, 243250 (2003).
[94] Erlicher, S., and P. Argoul, "Modal identification of linear non proportionally damped systems by wavelet transform," Mech. Syst. Signal Process. 21, 13861421 (2007).

[95] Daubechies, I., "Time frequency localization operators: A geometric phase space approach," IEEE Trans. Inf. Theory 34, 605612 (1988).

[96] Daubechies, I., and T. Paul, "Time frequency localisation operators a geometric phase space approach: II. The use of dilations," Inverse Probl. 4, 661680 (1988).

[97] Antoine, J. P., "The continuous wavelet transform in image process ing," CWI Quaterly 11, 323345 (1998).

[98] Olhede, S. C., and A. T. Walden, "Generalized Morse wavelets," IEEE. Trans. Signal Process. 50, 26612670 (2002).

[99] Antoine, J. P., R. Murenzi, P. Vandergheynst, and S. T. Ali, Two Dimensional Wavelets and their Relatives (Cambridge University, Cambridge, UK, 2004).

[100] Lilly, J. M., "Element analysis: A wavelet based method for analysing time localized events in noisy time series," Proc. R. Soc. A 473, 20160776 (2017).

[101] Bursac, P., G. Lenormand, B. Fabry, M. Oliver, D. A. Weitz, V. Viasnoff, J. P. Butler, and J. J. Fredberg, "Cytoskeletal remodelling and slow dynamics in the living cell," Nat. Mater. 4, 557561 (2005).

[102] Massiera, G., K. M. Van Citters, P. L. Biancaniello, and J. C. Crocker, "Mechanics of single cells: Rheology, time dependence, and fluctuations," Biophys. J. 93, 37033713 (2007).

[103] See supplemental material at https://doi.org/10.1122/1.5035127 for a more detailed presentation of the wavelet transforms and their implementation for singular signals. 